\title{
El ámbito normativo de la inteligencia interior en América Latina*
}

\section{0 âmbito informativo da inteligência doméstica na América Latina}

\section{The regulatory scope of domestic intelligence in Latin America}

JOSÉ MANUEL UGATE

Profesor de la aludida Universidad, de la Universidad Católica de Santiago del Estero, de la Universidad Abierta Interamericana, del Instituto Universitario de Seguridad Marítima, del Instituto Universitario de la Policía Federal Argentina, del Instituto de Inteligencia de las Fuerzas Armadas, y de la Escuela del Cuerpo de Abogados del Estado, así como miembro del Comité Académico del Instituto de Estudios Estratégicos de la Seguridad del Ministerio de Seguridad de la Nación y del Comité Académico del Instituto de Estudios de la Defensa "Manuel Belgrano" del Ministerio de Defensa Nacional Av. Alsina y Dalmacio Vélez Sársfield (4200), Santiago del Estero, República Argentina manuguart@gmail.com

RESUMEN En el trabajo, es objeto de análisis en una perspectiva comparada, el ámbito normativo correspondiente a la inteligencia interior en América Latina, cotejándolo con el existente en otros países, fundamentalmente países de significativa evolución institucional y caracterizados por una actividad de inteligencia con límites y control. La comparación evidencia la amplitud que posee la inteligencia interior en Latinoamérica, respecto de la que posee en los países utilizados para la comparación, basada tanto

* $\quad$ Artigo recebido em: 26/02/2012. Autor convidado. 
en la asignación legislativa de competencias sobre la base de conceptos muy amplios e incluso vagos, como, en ciertos casos, de la consideración de la actividad de inteligencia como una actividad ordinaria del Estado, que puede ser requerida para auxiliar a los responsables de la formulación de políticas en cualquier ámbito. El trabajo concluye proponiendo para Latinoamérica la adopción de conceptos más precisos y estrictos en materia de inteligencia interior, tanto en beneficio de la propia actividad - que podrá concentrar de ese modo sus medios humanos y materiales y sus recursos en aquellos aspectos fundamentales para el Estado - como de la vigencia de los derechos individuales.

Palabras-clave inteligencia, límites, legislación, derechos

ABSTRACT The purpose of this paper is to address domestic intelligence laws in force in Latin America from a comparative perspective. Such laws are compared with those in force in countries exhibiting significant institutional advancement in which the intelligence activity features limits and controls. The comparative analysis shows the all-embracing scope characteristic of domestic intelligence in Latin America versus that of the countries used as comparison, and that this is the result of both the legislative allocation of conceptually wide-ranging and even vague functions and, in some cases, the consideration of the intelligence activity as a regular activity of the State, likely to be required in aid of policy-makers in any area. The paper concludes proposing that Latin American countries adopt more precise and strict concepts of domestic intelligence for the benefit of both the activity itself -enabling it to concentrate its human and material means and resources to issues that are truly basic to the State- and the validity of individual rights.

Keywords intelligence, limits, legislation, rights

\section{¿Qué habremos de considerar? ¿Qué aspectos comprende la inteligencia interior en Latinoamérica?}

Delimitar el objeto de nuestro análisis constituye una preocupación inicial, que procuraremos solucionar.

Denominaremos, a los efectos del presente, inteligencia interior a la actividad de inteligencia que se realiza por parte de un Estado dentro de su propio territorio, dirigida hacia sus habitantes - tanto ciudadanos locales y residentes legales como extranjeros.

Por extensión, habremos de incluir dentro de este análisis a la contrainteligencia realizada por el Estado dentro de su propio territorio. tanto a aquélla que responde a concepciones clásicas de esta última actividad - contraespionaje, contrasabotaje, medidas de seguridad de contrainteligencia, en definitiva, actividad destinada a evitar la acción de organismos 
de inteligencia extranjeros e incluso de agentes de países extranjeros en el propio territorio, así como a proteger secretos industriales, comerciales y tecnológicos del país, como, en una concepción más amplia de aquélla, el contraterrorismo e incluso, en una perspectiva aún más amplia - sin que ello implique que compartamos tal perspectiva - y como establece la Ley № 25.520 de Inteligencia Nacional argentina (artículo $2^{\circ}$, numeral 2) en su definición de la contrainteligencia como: "la actividad propia del campo de la inteligencia que se realiza con el propósito de evitar actividades de inteligencia de actores que representen amenazas o riesgos para la seguridad del Estado Nacional", sin precisar la naturaleza de los actores, ni si son extranjeros, influidos por Estados u organizaciones extranjeras, o simplemente nacionales del país.

Asumimos que la inclusión a los fines de este trabajo de la contrainteligencia puede despertar polémicas, dado que tradicionalmente y en muchos países, este aspecto de la actividad de inteligencia hace referencia a la protección del propio país, sus habitantes e intereses, contra actividades de inteligencia - incluyendo operaciones encubiertas - de organismos de inteligencia extranjeros, organizaciones de fachada de éstos o agentes extranjeros clandestinos, o en ciertos países, de terrorismo internacional inspirado, financiado y apoyado desde el exterior, diferenciándose de la inteligencia interior (domestic intelligence), también denominada seguridad interna (internal security), destinada fundamentalmente a prevenir o contrarrestar intentos de derribar, debilitar o subvertir el sistema democrático, a lo que en algunos países se agrega el delito organizado, cuando tiene una entidad suficiente como para constituir una seria amenaza para el Estado.

No obstante, esta inclusión halla fundamento en la circunstancia de ser particularmente borrosos en Latinoamérica los límites entre la contrainteligencia y la inteligencia interior, dada la amplitud con que se concibe el concepto señalado en primer término; como también son especialmente borrosos en tales países los límites entre la actividad policial y uno de sus aspectos más importantes, la inteligencia criminal, y la actividad de inteligencia. Por tal razón, si excluyéramos la contrainteligencia del presente estudio, no tendríamos un panorama completo de la actividad de inteligencia que realizan los países latinoamericanos en el interior de sus países y respecto de sus propios habitantes - ciudadanos, extranjeros, y extranjeros residentes permanentes - dado que con contadas excepciones, y más allá de las denominaciones, el grueso de tal actividad no tiene como objeto la protección del país contra el accionar de los organismos de inteligencia extranjeros o los agentes de países foráneos, sino, en realidad, actividades realizadas por los propios nacionales. 
En su clásica obra Inteligencia estratégica para la política mundial estadounidense ${ }^{1}$ Sherman Kent se refirió a la "inteligencia de seguridad"2 (security intelligence) definiéndola como la inteligencia

relativa a la función de vigilancia o policía. Su misión es proteger a la nación y a sus miembros de los malhechores que trabajan procurando producir un daño nacional o internacional. En una de sus más dramáticas formas, es la información que trata continuamente de señalar a los agentes clandestinos enviados al país por potencias extranjeras. En otra, es la actividad que protege nuestras fronteras contra intrusos indeseables: personas entradas en forma ilegal, contrabandistas, distribuidores de drogas y demás. Identifica a los traidores nacidos en el país y a las personas que violan la ley federal. La inteligencia de seguridad es el conocimiento y la actividad que nuestras fuerzas defensoras del orden deben poseer antes de emprender una acción específica contra el malintencionado o el malhechor individual. ${ }^{3}$

Esta clásica definición de Shernan Kent no agota la actividad de inteligencia que muchos países, incluso de alto desarrollo institucional y que efectúan un preciso control de la actividad de inteligencia, realizan en su territorio. A ella debe agregarse la producción de inteligencia externa - referida a países u organizaciones externas - que realizan tales países - entre los que citaremos a Estados Unidos de América y Canadá, obteniendo información de tal naturaleza en sus propios territorios.

Cabe destacar inicialmente que aquellos países han sabido superar con mayor éxito el dilema representado por la contradicción entre algunas características del sistema republicano y democrático - "como la publicidad de los actos de gobierno, la responsabilidad de los gobernantes por sus actos, la transparencia en el accionar gubernamental" - y determinados aspectos de la actividad de inteligencia - como el secreto que rodea fuentes, métodos, identidades y operaciones, la utilización de fondos que poseen un sistema especial de rendición destinado a proteger el secreto, el empleo de un sofisticado instrumental tecnológico diseñado para penetrar la privacidad de los habitantes.

Así, ha sostenido Morton H. Halperin que

los requerimientos de las agencias de inteligencia y las normas de una sociedad abierta plantean el más agudo de los aparentes dilemas para un gobierno democrático. Las agencias de inteligencia, por su naturaleza, funcionan en secreto sin estar sujetas a las reglas normales del Estado. Una sociedad abierta, por

1 Utilizamos aquí una edición argentina, KENT, Sherman. Apéndice y Clase de Inteligencia. In: Inteligencia estratégica para la política mundial estadounidense. 4.ed. Buenos Aires: Editorial Pleamar, 1986, p.225-230.

2 Información de seguridad, en la traducción argentina.

3 KENT, Sherman. Apéndice, Clase de Inteligencia, p.225 y 230. 
otra parte, aborrece el secreto e insiste que todas las agencias gubernamentales deben cumplir plenamente con la Ley. ${ }^{4}$

Uno de los medios de superar tal dilema para los países aludidos consiste en diferenciar cuidadosamente la actividad de inteligencia que practican en su territorio y respecto de sus propios ciudadanos y residentes permanentes, de aquella que practican fuera de su territorio y respecto de los ciudadanos de otros países, no residentes.

Tal diferenciación tiene, según los países, criterios meramente territoriales, o bien territoriales-personales, incidiendo también en determinados casos el origen de la amenaza. No obstante, el criterio diferenciador fundamental suele ser el territorial.

Por las razones indicadas, habremos de referirnos aquí no sólo a la inteligencia interior o doméstica estrictamente, sino también de la inteligencia de seguridad en su conjunto, englobando en consecuencia tanto a la inteligencia interior, como a la contrainteligencia que tiene lugar en el propio territorio, para enfrentar amenazas externas - el que incluye contraespionaje, contrasabotaje, contraterrorismo internacional - comprendiendo, en consecuencia, tanto la actividad de inteligencia que realiza el Estado en su propio territorio y respecto de sus ciudadanos o residentes permanentes, como la que efectúa en dicho territorio respecto de amenazas externas, dirigidas directamente contra el país.

También habremos de incluir la obtención en el propio territorio de inteligencia sobre países, organizaciones o personas extranjeras, que aunque carezca de las características fundamentalmente defensivas que posee la inteligencia de seguridad, la circunstancia de ser obtenida en el propio territorio determina también la necesidad de límites a su respecto.

Habremos de adelantar que mientras en aquellos países que han logrado superar adecuadamente el dilema antes señalado, se produce la aludida diferenciación y el establecimiento de claros límites a la actividad de inteligencia en su territorio, ello no sucede en los países latinoamericanos, en los cuales la actividad de inteligencia se ejerce con idéntica extensión y características dentro del propio país y en el exterior (en este último caso, refiriéndonos a aquellos países que poseen capacidades de inteligencia exterior) sin diferenciarse en modo alguno entre ciudadanos propios y residentes legales y extranjeros, y, por sobre todo, con una amplitud muy significativa.

4 HALPERIN, Morton H. Intelligence in an open society. In: Intelligence: policy and process. Colorado: Boulder Westriew, 1985. 


\section{Criterios para la delimitación del ámbito a asignarse a la actividad de inteligencia y de las competencias de los órganos y organismos que la integran}

Abraham N. Shulsky ${ }^{5}$ detallando los diversos criterios existentes, con relación a la noción de inteligencia y a los ámbitos abarcados por ella, destacó que limitaba el significado de la palabra inteligencia al "ámbito tradicional, de información y actividades relativas a las preocupaciones de seguridad nacional de los gobiernos". Reconoció no obstante, que el término seguridad nacional era poco claro, pese a que su significado fundamental estaba relacionado con la protección de una nación contra amenazas, esencialmente militares, provenientes de países extranjeros.

Según Shulsky, la cuestión tenía mayor complejidad por la circunstancia de que los intereses de seguridad nacional y las amenazas contra tales intereses no podían ser considerados independientemente del tipo de gobierno que el país tenía y su perspectiva ideológica.

Obvio resulta, siguiendo esta reflexión, que mientras para muchos países, la expresión seguridad nacional comprende las amenazas contra el país provenientes de otros países extranjeros y aún del terrorismo internacional, para otros este término tiene un alcance mucho mayor, que incluye la protección de la seguridad interna contra amenazas surgidas de personas o grupos autóctonos, o incluso la protección de determinados valores culturales o ideológicos, y, en una visión extrema - propia de los regímenes autocráticos - la protección del propio gobierno, no ya contra atentados o ataques ilegales, sino contra toda persona o grupo que se proponga sustituirlo, aún cuando fuera por medios legales, ya sea electorales - en el caso de existir - o aún a través del disenso pacífico. Así, como sostiene Shulsky ${ }^{6}$ "cómo define un gobierno esas amenazas internas depende fuertemente del tipo de gobierno que es".

Para los países con sistemas democráticos consolidados, la oposición política legal y, en general, las actividades políticas, gremiales, asociativas o religiosas legítimas no constituyen objeto de la actividad de inteligencia.

Los límites a la actividad de inteligencia no son iguales en todos los países que los establecen.

Muchos de los países con fuerte tradición democrática distinguen entre inteligencia interior e inteligencia exterior, asignando cada una de tales funciones a un organismo diverso, caracterizándose el primero por tener competencias cuidadosamente limitadas, mientras que el segundo, aún cuando no carece de una clara especificación de competencias, suele presentar mayor amplitud.

\footnotetext{
SHULSKY, Abraham N. Silent warfare: understanding the world of intelligence. Virginia: Brassey's, 1991.

SHULSKY, Abraham N. Silent warfare. Sobre los diversos significados que puede tener la expresión Seguridad Nacional según las características del régimen político imperante. UGARTE, José Manuel de. Los conceptos jurídicos y políticos de la seguridad. Buenos Aires: Editorial Plus Ultra S.A., 2005.
} 
Tal distinción entre conflictos externos e internos, a los fines de la atribución de competencias a los organismos de inteligencia, tiene lugar, en el caso de ciertos países, con un criterio fundamentalmente territorial, como sucede en el caso de la competencia de la Dirección Central de Inteligencia Interior de la República Francesa. En otros casos, se emplea un criterio personal-territorial, como puede advertirse en el caso de Estados Unidos de América.

Ya sea que se atribuya tal distinción a la consideración de la diferente naturaleza de los conflictos que afronta un Estado como tal con otros Estados, en el marco del sistema internacional, con relación a aquellos producidos en el seno de la misma unidad política ${ }^{7}$ o bien a la diversidad de situaciones representada por la actividad de inteligencia dentro del propio territorio - donde rige la ley del Estado y existe un orden coactivo único, respecto del exterior, donde ello no sucede - o a ambas circunstancias- lo cierto es que la vigencia de la aludida distinción y, a la vez, la existencia de claros límites en materia de inteligencia interior se da en la casi totalidad de los países con alto nivel de institucionalidad.

Esta distinción posibilita la aplicación a los conflictos internos de criterios diversos a los empleados respecto de los externos; o bien, aplicar a la seguridad interior, criterios y métodos diversos a los utilizados en materia de defensa y política exterior.

En los países que poseen un adecuado grado de control de sus órganos de defensa, seguridad pública e inteligencia, y en los cuales se han logrado significativos avances en la no sencilla tarea de obtener eficacia específica en tales materias y, al propio tiempo, salvaguardar adecuadamente los derechos y garantías individuales de sus ciudadanos, puede advertirse que el esfuerzo fundamental en materia de inteligencia tiene lugar hacia el exterior del propio país, concebido como una unidad; mientras que en el interior del país la actividad de inteligencia tiene límites mucho más estrictos que los existentes en materia de inteligencia exterior y, por otra parte, está dirigida en forma predominante hacia aquellas amenazas que, aunque se manifiestan en el propio país, provienen del exterior de éste.

En estos países, el objeto de la actividad de inteligencia es la defensa del Estado y no de determinado régimen o gobierno. Por ello, en el ámbito interno, las amenazas que pueden dar lugar al accionar de la inteligencia, claramente determinadas, son las amenazas contra el Estado en sí o contra el sistema de gobierno libremente electo, o contra su legítimo desempeño. No se dirige la actividad de inteligencia contra los opositores políticos legales del gobierno, ni contra la legítima actividad política, asociativa, sindical, religiosa, etc.

7 Hemos sostenido este punto de vista en UGARTE, José Manuel de. Seguridad interior. Buenos Aires: Fundación Arturo Illia, 1990. 
Un ejemplo paradigmático de estas características está representado por la disposición contenida en el artículo 7 de la Ley del $1^{\circ}$ de abril de 1981, número 121, Nuevo ordenamiento de la Administración de la Seguridad Pública de la República Italiana, que establece que:

Está prohibida en todo caso la recogida de informaciones y datos sobre los ciudadanos por el solo hecho de su raza, fe religiosa u opinión política, o de su adhesión a principios de movimientos sindicales, cooperativos, asistenciales, culturales, así como por la legítima actividad que desarrollen como pertenecientes a organizaciones que actúen legalmente en los sectores antes indicados.

Entre las características que hemos señalado, se encuentra aquella relativa a que, en los sistemas democráticos, la inteligencia interior - y, por extensión, la contrainteligencia que se realiza en el propio país - es esencialmente limitada y dirigida contra las reales amenazas contra la seguridad del Estado y la vigencia del sistema democrático.

Tal carácter reconoce una razón: la actividad de inteligencia no constituye una actividad común u ordinaria del Estado democrático, aunque tenga carácter permanente e importancia fundamental para la seguridad del Estado y de las instituciones democráticas. ${ }^{8}$

No constituye una actividad ordinaria o común del Estado democrático, precisamente por sus antedichas características.

En definitiva, nos encontramos, entonces, frente a una paradoja: la actividad de inteligencia ocasiona inocultables peligros al sistema democrático, pero los países democráticos para subsistir y aún el propio sistema democrático a menudo necesita de la actividad de inteligencia en sentido amplio ${ }^{9}$ para defenderse de acechanzas, aún internas, que pretenden destruirlo.

Como consecuencia del reconocimiento en los países democráticos, tanto de la necesidad, como de la preocupación antedichas, es que la actividad de inteligencia reviste características diferentes en un Estado democrático y en un Estado totalitario.

Permítasenos plantear una hipótesis: En un Estado democrático, la actividad de inteligencia es, esencialmente, limitada, en lo que se refiere al interior del país. Puede, pues, discutirse si la intervención del Estado en la economía va a tener menor o mayor alcance, y la solución puede variar según las épocas y los conceptos predominantes; o bien, la mayor o menor magnitud de las prestaciones económico-sociales que va a brindar a sus habitantes. En cambio, no parece discutible que una actividad de inteligencia con competencias ilimitadas dentro del propio país no es compatible con la real vigencia del sistema democrático.

8 Hemos expresado este punto de vista en el artículo, UGARTE, José Manuel de. Sistemas de inteligencia y democracia. In: LEÓN, Bernardo Arévalo de. Función militar y control democrático. El Amanuense: UNOPS, 2001.

En sentido amplio, por comprender a la contrainteligencia y a la inteligencia interior. 
¿Cómo establecer esa limitación? Básicamente, considerando en primer lugar la necesidad de distinguir entre la actividad de inteligencia en el exterior y el interior del propio Estado.

En el interior del propio Estado, donde postulamos la necesidad de establecer el carácter limitado antedicho, el objeto de la actividad de inteligencia - empleando esta expresión en sentido amplio, que incluye a la contrainteligencia - debe estar constituido por las concretas amenazas a la seguridad del Estado, provocadas por actividades - no pensamientos o creencias - dirigidas contra él por países extranjeros, o por organizaciones extranjeras o nativas que procuren atentar contra el Estado, cambiar por medios ilegales su sistema de gobierno, o atentar contra las autoridades del país o el legítimo ejercicio por parte de éstas de sus atribuciones, por organizaciones terroristas, o bien por organizaciones criminales que hubieran adquirido un desarrollo tal, que representara un serio peligro para la seguridad del Estado.

Es decir, que la actividad de inteligencia, dentro del propio país no es una actividad ordinaria del Estado democrático. Es una actividad esencialmente limitada de dicho Estado, reservada, con un criterio restrictivo, a aquellas amenazas susceptibles de destruir al Estado o al sistema democrático.

La actividad de inteligencia no reemplaza a un órgano estatal de difusión o de relaciones públicas, ni a la comunidad académica, ni a los órganos de realización de encuestas, tanto estatales como privados, ni a la diplomacia, ni a los órganos de gestión de comercio internacional, ni a la policía.

La actividad de inteligencia no puede tener la misma amplitud, cuando se proyecta hacia el exterior, que dentro del propio país. Aún sin tener una expansión incontrolada y bajo el control cuidadoso de la política exterior, a la cual inexorablemente debe estar subordinada, la actividad de inteligencia puede y debe escudriñar la situación de aquellos países extranjeros de interés para la política exterior, económica y de defensa del país, procurando advertir sobre posibles amenazas de cualquier tipo - políticas, económicas y militares - y también oportunidades políticas y económicas: alianzas convenientes, posibilidades de creación de espacios comerciales integrados etc.

Se trata de una actividad que puede poseer cierta amplitud cuando se proyecta hacia el exterior del propio país para proveer a su defensa e incluso, buscando oportunidades políticas, económicas y tecnológicas. Pero hacia adentro, requiere cuidadosa y estricta limitación.

De allí la importancia que poseen en esta materia las competencias. Las competencias, en materia de inteligencia, debieran hallarse cuidadosamente delimitadas en leyes públicas, no, obviamente, en normas secretas.

Hemos postulado precedentemente que en un Estado democrático, y en el interior del la actividad de inteligencia es esencialmente limitada.

Dicho carácter no hace referencia, ciertamente, a que constituya una actividad episódica. Es permanente; pero debiera ser reservada - muy 
particularmente en el interior del país - a aquellas situaciones que implican un real compromiso, una amenaza a la seguridad del Estado, al sistema democrático, a las autoridades, al legítimo ejercicio por parte de éstas de sus atribuciones.

En el otro extremo, nos encontramos con la actividad de inteligencia ilimitada. La actividad de inteligencia, desde esta perspectiva, constituiría una mera herramienta de obtención de información del Estado y fundamentalmente del gobierno, quien no encontraría cortapisas tampoco para influir en su beneficio a la opinión pública, utilizando las estructuras secretas y los fondos reservados que se hallan a su disposición. Toda información que sea de interés para la gestión gubernamental, puede ser obtenida a través de esta herramienta, sea en el exterior o en el interior del país, de nacionales o extranjeros. Idéntica libertad regiría para operaciones de inteligencia.

¿Cómo se establecen límites precisos a la inteligencia doméstica?

En primer lugar, estableciendo en la legislación con claridad la competencia de los organismos de inteligencia facultados para actuar en el propio país - y respecto de los propios ciudadanos o residentes permanentes - limitando tal competencia a amenazas concretas y actuales para la seguridad del Estado y del sistema democrático.

En segundo lugar, estableciendo prohibiciones a los organismos de inteligencia relativas a la realización de determinadas actividades.

Examinaremos aquí algunos casos de inteligencia con límites, para luego centrarnos en el análisis, desde este punto de vista, de la actividad de inteligencia en América Latina.

\section{La actividad de inteligencia en el propio territorio con límites Estados Unidos de América}

El caso de Estados Unidos de América reviste particular interés, dada la muy significativa expansión de la actividad de inteligencia en el territorio estadounidense que siguió a los atentados del 11 de septiembre de 2001, con consecuencias como la sanción de la Ley PATRIOT del 2001, la creación del Departamento de Seguridad Interna a través de la Ley de Seguridad Interna del 2002, la sanción de la Ley de Reforma de Inteligencia y Prevención del Terrorismo de 2004, y muy especialmente, los importantes cambios producidos en la Federal Bureau of Investigation (FBI). FBI és un organismo con facultades policiales que unió a sus ya conocidas actividades y capacidades de investigación de delitos federales no asignados en exclusividad a otro organismo - la inteligencia interior - dirigida contra personas $u$ organizaciones internas que pretenden atentar contra el sistema democrático o imponer ideas por medios ilegales - y la contrainteligencia y contraterrorismo internacional, la obtención de inteligencia externa en 
territorio estadounidense e incluso inteligencia antiproliferación de armas de destrucción masiva.

Se deben destacar, en el sentido indicado, a) la creación de la Rama de Seguridad Nacional (National Security Branch) (12 de septiembre de 2005) y dentro de ella, la Dirección de Inteligencia (Intelligence Directorate), b) la creación de los Grupos de Inteligencia de Campo (Field Intelligence Groups-F/Gs), órganos establecidos en cada una de las 56 Oficinas de Campo (Field Offices) del FBI, compuestos fundamentalmente por agentes especiales - escalafón fundamental de la FBI, integrado por funcionarios con facultades policiales y formación que incluye aspectos legales, aptitud investigativa y de inteligencia, con diversas especialidades - y personal profesional que incluye analistas de inteligencia, lingüistas y especialistas en vigilancia, con la misión de ejecutar actividades de inteligencia de campo bajo la dirección de los órganos centrales del FBI; c) el dictado por parte del Procurador General (Attorney General) ${ }^{10}$ de las Directivas para Operaciones Domésticas de la FBI, que unificando directivas anteriormente vigentes para diversos aspectos, incluyó las investigaciones criminales, las investigaciones de seguridad nacional - incluyendo contrainteligencia y contraterrorismo - y la obtención en territorio estadounidense de inteligencia externa, unificando hasta el límite de lo posible los estándares para la realización, autorización y control de estas actividades; d) la implementación de la cooperación de las instituciones policiales federales, estatales, de condado, locales y tribales para compartir inteligencia criminal y aportar información útil para la defensa del país contra el terrorismo, a partir de instrumentos como el Plan Nacional para Compartir Inteligencia Criminal (2003), e) la creación de Centros de Fusión etc.

Frente a estas nuevas realidades, representativas de notables cambios, realizados virtualmente en cinco años, cabe preguntarnos: ¿Carece de límites la actividad de inteligencia dentro del territorio estadounidense?

La respuesta inicial es que continúan existiendo en Estados Unidos de América múltiples distinciones en materia de inteligencia entre su realización dentro del territorio nacional y respecto de personas estadounidenses ciudadanos y residentes legales - y en el exterior y respecto de extranjeros.

Esas distinciones vienen desde los orígenes de la actual Comunidad de Inteligencia estadounidense.

Cabe recordar así entre ellos, en primer lugar, a la Agencia Central de Inteligencia, fiel expresión - al menos inicialmente - del paradigma de Sherman Kent, y que tuvo desde su nacimiento en la Ley de Seguridad Nacional de 1947, conforme al pensamiento de su inspirador, una importante

10 Funcionario estadounidense, con formación legal, que reúne las facultades de Ministro de Justicia, cabeza del Ministerio Público, jefe de la abogacía estatal y órgano de dictamen jurídico de máximo nivel, y de quien dependen organismos con facultades policiales e investigativas de suma importancia, como la ya referida FBI, la Administración para la aplicación coactiva de la ley en materia de drogas (Drug Enforcement Administration - DEA), la Oficina de Alcohol, Tabaco, Armas de Fuego y Explosivos (ATF), entre otras. 
limitación: aún en su versión actual ${ }^{11}$ la referida ley, incorporada al Código de Estados Unidos, continúa estableciendo que el Director de dicho organismo: "colectará inteligencia a través de fuentes humanas y por otros medios apropiados, excepto que no tendrá facultades policiales ni de citación compulsiva, o funciones de seguridad interna". ${ }^{12}$

Un breve examen de las características actuales de la Comunidad de Inteligencia estadounidense permite advertir - conforme a la normativa vigente - fundamentalmente, Código de los Estados Unidos, Título 50, Capítulo 15, Subcapítulo I, parágrafos 402 a 404o-2, y la Orden Ejecutiva N012.333 y sus modificaciones efectuadas en los años 2003, 2004 y 2008, una conducción y coordinación del Sistema a cargo del ya referido Director de Inteligencia Nacional, sujeto a la autoridad, dirección y control del Presidente - asistido a su vez en el ejercicio de tales facultades por el Consejo de Seguridad Nacional-; y la existencia de un organismo de inteligencia exterior, la Agencia Central de Inteligencia, que obtiene y analiza inteligencia externa, realiza actividades de contrainteligencia - referidas a amenazas de origen externo - y sin cumplir funciones de seguridad interna, lleva a cabo operaciones encubiertas aprobadas por el Presidente ${ }^{13}$ y tiene a su cargo - siempre bajo las directivas del ya referido Director - las vinculaciones con organismos de inteligencia de otros países y con organizaciones internacionales. Preciso es recordar que tales operaciones encubiertas no pueden ser llevadas a cabo - conforme tanto a la Orden Ejecutiva referida, como al Código de los Estados Unidos - con el propósito de influir los procesos políticos de los Estados Unidos, sus procesos políticos, opinión pública, políticas, o los medios de comunicación. La aludida Agencia Central tiene la coordinación de la obtención clandestina de inteligencia externa a través de fuentes humanas o a través de medios manejados por personas y de las actividades de contrainteligencia fuera de los Estados Unidos.

Importa señalar, además, que las políticas y los procedimientos para la coordinación de actividades de contrainteligencia y para la obtención clandestina de inteligencia externa dentro del territorio estadounidense están sujetos a la aprobación del ya aludido Procurador General (Attorney General). En otras palabras, es el funcionario abogado de mayor rango en Estados Unidos quien debe supervisar las actividades de inteligencia y contrainteligencia que se llevan a cabo en territorio estadounidense.

Estados Unidos cuenta asimismo en su vasto y complejo sistema de inteligencia, además de los órganos y organismos ya nombrados, con un organismo de inteligencia de Defensa, la Agencia de Inteligencia de Defen-

11 U.S.A. National Security Act of 1947, chapter 343, 61 Stat. 496, approved July 26, 1947. As amended through PL 110-53, enacted August 3, 2007.

12 U.S.A. Código de los Estados Unidos 403-4a, sección 104A, apartado 50, párrafo (d), numeral (1)

13 Recordemos, para lectores poco familiarizados con esta temática, que el concepto de operaciones encubiertas (covert operations) comprende actividades relacionadas para influir la situación política, económica o militar de países extranjeros, sin que se advierta el origen o patrocinio de la actividad, constituyendo de ese modo un recurso intermedio entre la diplomacia y la guerra. 
sa, con un organismo de inteligencia de señales - la Agencia de Seguridad Nacional - una Oficina Nacional de Reconocimiento encargada de la investigación, desarrollo, adquisición, lanzamiento, despliegue y operación de sistemas de reconocimiento aéreo para obtener información e inteligencia, y una Agencia Nacional de Inteligencia Geoespacial, encargada de obtener, procesar, analizar, producir y diseminar inteligencia geoespacial y datos para propósitos de inteligencia y contrainteligencia. Estos organismos fundamentalmente obtienen inteligencia externa. También cuenta con los elementos de inteligencia de las Fuerzas Armadas, que obtienen información y producen, analizan y diseminan inteligencia y contrainteligencia de defensa y relacionada con la defensa, fundamentalmente táctica o de combate.

Existen otros órganos de inteligencia ${ }^{14}$ tales como la Oficina de Inteligencia e Investigación del Departamento de Estado, la Oficina de Inteligencia y Análisis del Departamento del Tesoro, la Oficina de Inteligencia y Análisis del Departamento de Seguridad Interna, la Oficina de Inteligencia de Seguridad Nacional de la Administración de Aplicación Coactiva de la Ley en Materia de Drogas, la y la Oficina de Inteligencia y Contrainteligencia del Departamento de Energía, facultados para obtener información de fuentes abiertas, analizar, producir y diseminar información e inteligencia para apoyar misiones nacionales y de sus respectivos departamentos.

También la Oficina del Director de Inteligencia Nacional tiene facultades similares, para apoyar las misiones de la referida oficina, incluyendo el Centro Nacional de Contraterrorismo, y para apoyar otras misiones nacionales. Pero preciso es tener en cuenta que el referido Director tiene acceso a toda la información e inteligencia relevante a la seguridad nacional o requerida para el cumpliendo de las amplias facultades de conducción y coordinación del Sistema estadounidense, lo que ciertamente brinda amplias posibilidades para el cumplimiento de las funciones de la referida Oficina y para el aludido Centro Nacional de Contraterrorismo.

También el Departamento de Seguridad Interna, además de la actividad y facultades propias de su Oficina de Inteligencia y Análisis, está facultado a través del Servicio Secreto de Estados Unidos a realizar actividades tendientes a determinar la existencia y las capacidades de equipo de vigilancia utilizado contra el Presidente o el Vicepresidente, la Oficina Ejecutiva del Presidente, y otras autoridades respecto de las cuales exista autorización brindada por el Secretario de Seguridad Interna.

Asimismo, el Departamento de Energía, además de las facultades ejercidas por su Oficina de Inteligencia y Contrainteligencia a que hemos hecho referencia precedentemente, provee capacidades expertas en ma-

14 En el presente trabajo, denominados organismo de inteligencia (intelligence service) a los organismos técnicos especializados en inteligencia, capaces de cumplir todas las funciones propias del ciclo de la inteligencia, y órganos de inteligencia, a aquellos órganos con capacidad de cumplir alguna o algunas de tales funciones - habitualmente, análisis de información obtenida por organismos de inteligencia - que generalmente integran departamentos ministeriales $u$ otros organismos con funciones diversas a la inteligencia. 
teria científica, técnica y analítica a otros organismos, participando incluso en la formulación de requerimientos para la obtención de información y de análisis en el caso en que sus capacidades científicas y técnicas puedan contribuir a ello, entre otros aspectos.

Hemos dejado para el final a un organismo realmente clave en materia de inteligencia y contrainteligencia en territorio estadounidense: la ya referida $\mathrm{FBI}$.

Ya hemos aludido anteriormente al significativo desarrollo de las capacidades de inteligencia de la FBI En la actualidad y como consecuencia fundamental de los atentados del 11 de septiembre de 2001, la obtención de inteligencia externa dentro del territorio estadounidense constituye una de las fundamentales actividades de este organismo, sumándose a las tradicionales contrainteligencia, contraterrorismo, seguridad interna e investigación criminal.

La FBI posee una característica que la diferencia de otros organismos de inteligencia interior y que le brinda sus características casi únicas - con pocas excepciones entre las que se cuenta la actual Dirección Central de la Seguridad Interior francesa, organismo de contrainteligencia y seguridad interna francés, que constituye una de las Direcciones Generales de la Dirección Nacional de la Policía Nacional francesa - que es su integración como escalafón básico, por agentes especiales, funcionarios con facultades policiales - investigar delitos y arrestar delincuentes, entre otras - poseyendo como hemos señalado un escalafón profesional de analistas, especialistas en lenguaje, y especialistas en vigilancia, entre otros. ${ }^{15}$

Pese a esta integración - característica de un organismo con funciones policiales- acumula, como ya se ha señalado, funciones de investigación criminal, de inteligencia, contrainteligencia y seguridad interna. Por ello, como bien se señala en las ya citadas directivas, la FBI es un organismo de inteligencia así como un organismo policial. ${ }^{16}$

Aún cuando esta circunstancia podría teóricamente determinar riesgos institucionales en un país que no tuviera la tradición de respeto de las libertades civiles de sus ciudadanos que posee Estados Unidos, lo cierto es que todas las actividades señaladas, que se realizan, como hemos señalado, bajo la dependencia orgánica del máximo funcionario legal estadounidense - sin perjuicio de la dependencia funcional que en materia de inteligencia existe respecto del Director de Inteligencia Central - están sujetas a un elaborado sistema de autorizaciones y controles previstos en las ya citadas Directivas del Procurador General para Operaciones Domésticas de la FBI,

15 Existen ejemplos de alguna similitud en este sentido, como el recientemente suprimido Departamento Administrativo de Seguridad de Colombia, y el Servicio Bolivariano de Inteligencia Nacional (SEBIN) venezolano - antigua Dirección de los Servicios de Inteligencia y Prevención - DISIP- que constituyen organismos de inteligencia con integrantes que poseen también facultades policiales. No obstante, estos organismos constituyen ejemplos de los riesgos ínsitos en este esquema.

16 U.S.A. FBI. Attorney general guidelines for domestic FBI operations. Washington D.C., p.9. 
que distinguen distintos tipos de investigaciones, detallan los medios que pueden emplearse para cada una de ellas y los requisitos autorizatorios y de control que rigen en cada caso.

Se prevé que todas las operaciones previstas por las Directivas en examen - que, recordemos, se refieren a operaciones llevadas a cabo en territorio estadounidense -deben ser llevadas a cabo de un modo congruente con todas las leyes, reglamentos y políticas aplicables, incluyendo aquellas que protegen la privacidad y las libertades civiles.

Se destacan el deber y la facultad de supervisión de la División Seguridad Nacional del Departamento de Justicia, de la División de Inspección de la FBI, de la Oficina del Consejero General y de la Oficina de Integridad y Cumplimiento de dicho organismo, imponiéndose particularmente a la Sección de Supervisión de la División Seguridad Nacional del Departamento de Justicia, conjuntamente con la Oficina del Consejero General de la FBI, la obligación de llevar a cabo revisiones regulares de todos los aspectos de las operaciones en materia de seguridad nacional y de inteligencia externa llevadas a cabo por la FBI.

Se requiere además que ante métodos investigativos de igual eficacia, debe optarse por el menos intrusivo ${ }^{17}$ previéndose además que el uso de las facultades y métodos previstos en las Directivas, tendrá por finalidad la protección de Estados Unidos y de su pueblo de delitos que involucren violación de leyes federales y amenazas a la seguridad nacional, así como para cumplir los objetivos estadounidenses en materia de inteligencia externa. ${ }^{18}$

Mientras las evaluaciones no tienen requisitos especiales de aprobación por involucrar el empleo de medios poco intrusivos, fundamentalmente fuentes públicas o archivos o registros federales, las investigaciones basadas requieren una base -alegaciones, informes, hechos o circunstancias indicativas de posible actividad criminal o lesiva de la seguridad nacional, o el potencial de obtener información útil para responder a requerimientos de inteligencia externa, requiriendo su iniciación aprobación supervisora. Según la importancia de la base con la que se cuenta, podrán iniciarse investigaciones preliminares - con medios limitados y un límite temporal - o investigaciones plenas, en las que se pueden emplear todos los métodos investigativos legales.

Se trata, en definitiva, de distinciones, límites y controles que se aplican tanto a la actividad de investigación del delito y de inteligencia criminal, como a la contrainteligencia y a la producción de inteligencia externa realizada por la FBI en territorio estadounidense.

Para concluir respecto de Estados Unidos de América en la materia que nos ocupa, habremos recurrir a un elemento fundamental: la Orden Ejecutiva 12.333 sobre Actividades de Inteligencia en Estados Unidos, que estableció

17 U.S.A. FBI. Directivas del procurador general para operaciones domésticas de la FBI, p.12, punto C apartado 2. 18 U.S.A. FBI. Directivas del procurador general para operaciones domésticas de la FBI, p.12, punto C apartado 1. 
normas fundamentales y la división de competencias entre los organismos de inteligencia en Estados Unidos, por las Órdenes Ejecutivas 13284; 2003, 13355; 2004 y 13470; 2008. Al respecto, debemos señalar el texto de la Orden Ejecutiva aludida continúa estableciendo distinciones según la actividad de inteligencia - o de contrainteligencia - tenga lugar dentro o no del territorio estadounidense, así como, fuera del territorio estadounidense, respecto de personas estadounidenses (ciudadanos estadounidenses 0 residentes legales en el país) o no.

Así, el punto 1.3 relativo a las facultades del Director de Inteligencia Nacional, apartado B) numeral 20 - facultad de asegurar la superación de los conflictos, la integración y la coordinación de las actividades de inteligencia llevadas a cabo por elementos de la Comunidad de Inteligencia o financiadas por el Programa de Inteligencia Nacional, establece que ello debe tener lugar conforme a las siguientes políticas y procedimientos:

El Director de la Oficina Federal de Investigación coordinará la obtención clandestina de inteligencia externa obtenida a través de fuentes humanas o de medios activados por humanos y actividades de contrainteligencia dentro de los Estados Unidos; B) El Director de la Agencia Central de Inteligencia coordinará la obtención clandestina de inteligencia externa obtenida a través de fuentes humanas o de medios activados por humanos y actividades de contrainteligencia fuera de Estados Unidos. C) Todas las políticas y los procedimientos para la coordinación de actividades de contrainteligencia y la obtención clandestina de inteligencia externa dentro de Estados Unidos estará sujeta a la aprobación del Procurador General [máximo funcionario legal de Estados Unidos, equivalente al ministro de Justicia y al jefe del Ministerio Público de otros países].

Asimismo, surge del punto 2.3 Obtención de Información, que los elementos de la Comunidad de Inteligencia estadounidense están autorizados para obtener, retener o diseminar información relativa a personas estadounidenses:

Ciudadanos estadounidenses o residentes legales en el país (...) sólo de acuerdo con procedimientos establecidos por el jefe del elemento respectivo de la Comunidad de Inteligencia o por el jefe de un departamento que contenga tal elemento, y aprobados por el Procurador General, de acuerdo con las facultades otorgadas por la Parte 1 de esta Orden, después de consultar con el Director [de Inteligencia Nacional].

Puede así advertirse que la obtención relativa a personas estadounidenses sólo puede ser obtenida conforme a procedimientos que requieren la aprobación del máximo funcionario legal del país.

Se establece además que tales procedimientos deben establecer - entre otros aspectos - que la 
b) obtención dentro de los Estados Unidos de inteligencia externa no susceptible de ser obtenida de otra manera será llevada a cabo por la Oficina Federal de Investigación (FBI) o, cuando es buscada significativa inteligencia externa, por otros elementos autorizados de la comunidad de inteligencia, quedando establecido que no puede ser obtenida inteligencia externa por tales elementos para el propósito de adquirir información relativa a las actividades domésticas de personas estadounidenses.

\section{Asimismo, en materia de}

e) información necesarias para proteger fuentes, métodos y actividades de inteligencia o contrainteligencia externas de revelación no autorizada (...) se prevé que (...) la obtención dentro de Estados Unidos será llevada a cabo por la Oficina Federal de Investigación (FBI), excepto que otros elementos de la Comunidad de Inteligencia pueden obtener la información relativa a empleados actuales o anteriores, contratistas actuales o anteriores, o sus empleados actuales o anteriores, o postulantes a tales empleos o contratos.

También se faculta a obtener "h) información obtenida por reconocimiento aeroespacial no dirigido a personas estadounidenses específicas".

Asimismo, en material de Técnicas de Obtención, el punto 2.4 prevé que

los elementos de la Comunidad de Inteligencia usarán las técnicas de obtención menos intrusivas posibles dentro de los Estados Unidos o dirigidas contra personas estadounidenses en el extranjero.

Elementos de la Comunidad de Inteligencia no están autorizados para utilizar técnicas tales como vigilancia electrónica, búsquedas físicas no consentidas, vigilancia de correo, vigilancia física, o aparatos de monitoreo, a menos que ese uso tenga lugar conforme con procedimientos establecidos por el jefe del elemento respectivo de la Comunidad de Inteligencia o el jefe de un departamento que contenga tal elemento y aprobados por el Procurador General, luego de consultar con el Director. Tales procedimientos protegerán los derechos constitucionales y legales y limitarán el uso de tal información a propósitos legales gubernamentales, y no autorizarán:

a) a la Agencia Central de Inteligencia, a empeñarse en vigilancia electrónica dentro de los Estados Unidos, excepto por el propósito de adiestramiento, testeo, o ejecución de contramedidas ante vigilancia electrónica hostil;

b)Búsquedas físicas no consentidas, en Estados Unidos, por elementos de la Comunidad de Inteligencia que no sean la Oficina Federal de Investigación (FBI), excepto para 1) Búsquedas por elementos de contrainteligencia de las fuerzas armadas dirigidas contra personal militar dentro de Estados Unidos o en el extranjero para propósitos de inteligencia, cuando fueran autorizadas por un comandante militar facultado para aprobar búsquedas físicas para propósitos de aplicación de la ley, basados en una conclusión de causa probable para creer que tales personas están actuando como agentes de potencias extranjeras; y 2) Búsquedas por parte de la CIA de propiedades personales de personas no estadounidenses legalmente en su posesión;

c) Vigilancia física de una persona estadounidense en los Estados Unidos por elementos de la Comunidad de Inteligencia diversos a la Oficina Federal de 
Investigación, excepto para 1) Vigilancia física de actuales o anteriores empleados, actuales o anteriores contratistas del elemento de inteligencia o de sus actuales o anteriores empleados, o solicitantes de tal empleo o contratación; y 2) Vigilancia física de una persona militar empleada por un elemento que no fuera de inteligencia, de una fuerza armada; y

d) Vigilancia física de una persona estadounidense en el extranjero para obtener inteligencia externa, excepto para obtener información que no pueda ser razonablemente obtenida por otros medios.

\section{Asimismo, se faculta al Procurador General (punto 2.5) para}

aprobar el uso para propósitos de inteligencia, dentro de los Estados Unidos o contra una persona estadounidense en el extranjero, de cualquier técnica para la cual un mandamiento (warrant) sería requerido si fuera utilizada para propósitos policiales, quedando establecido que tales técnicas no serán utilizadas a menos que el Procurador General haya determinado en cada caso que hay una causa probable para creer que tal técnica está dirigida contra una potencia extranjera o un agente de una potencia extranjera. La facultad delegada conforme a este parágrafo, incluyendo la facultad de aprobar el uso de vigilancia electrónica conforme está definida en la Ley de Vigilancia para Inteligencia Externa de 1978, y sus modificaciones, será ejercida de acuerdo con dicha ley.

Tales distinciones, así como el correlativo requerimiento de la aprobación del máximo funcionario legal con relación a procedimientos que involucran obtención de información en el propio territorio o respecto de personas estadounidenses, o la asignación de facultad de coordinación y de competencia prioritaria para obtención de información y realización de la contrainteligencia dentro del territorio estadounidense a la Oficina Federal de Investigación, permiten determinar la existencia de las ya referidas diferencias y controles en lo relativo a la actividad de inteligencia en territorio estadounidense, particularmente respecto a las personas estadounidenses.

Finalmente, en materia de Objetivos del Esfuerzo de Inteligencia, la Parte I numeral 1.1 de la referida Orden Ejecutiva establece que

el esfuerzo de inteligencia estadounidense proveerá al Presidente, al Consejo de Seguridad Nacional y al Consejo de Seguridad Interna con la información necesaria en la cual basar decisiones relativas a la conducción de las políticas exterior, de defensa y económica, y la protección de los intereses nacionales de Estados Unidos de amenazas externas a la seguridad.

De lo expuesto, cabe deducir que ni las cuestiones de política interna del país, ni las actividades lícitas que desarrollen las personas estadounidenses, pueden constituir objetivo válido de la actividad de inteligencia estadounidense.

Por ello, cabe concluir que pese a la significativa expansión que tuvo con posterioridad al 11 de septiembre de 2001, la actividad de inteligencia 
estadounidense en el territorio de su país tiene claros límites, así como controles que se encuentran entre los más amplios del mundo. ${ }^{19}$

\section{Canadá}

Uno de los ejemplos más interesantes de delimitación cuidadosa de la actividad de inteligencia en el interior del país, es el representado por la Ley del Servicio Canadiense de Inteligencia de Seguridad de 1984.

Ese cuerpo legal surgió de la desafortunada experiencia del Security Service (Servicio de Seguridad), creado en Canadá dentro de la Real Policía Montada de Canadá, con asistencia de su homónimo británico.

Las irregularidades puestas de manifiesto por las investigaciones realizadas por la Real Comisión Investigadora sobre Ciertas Actividades de la Real Policía Montada del Canadá encabezadas por el Juez David Cargill Mc Donald (1977-1981), que entre otros aspectos evidenció los riesgos institucionales de la asignación de la actividad de contrainteligencia e inteligencia interior a un organismo policial.El juez determinó la sanción de la Ley del Servicio Canadiense de Inteligencia de Seguridad de 1984, con la creación del aludido servicio, con competencias de contrainteligencia, inteligencia interior y de inteligencia exterior dentro del territorio de Canadá, privado de toda facultad policial y con muy significativos controles externos e internos. ${ }^{20}$

Conforme estableció la sección 12 de la ley referida, relativo a Deberes y funciones del Servicio, en lo relativo a la función fundamental de este organismo - sin perjuicio de otras funciones secundarias, entre las que se cuentan la provisión al Gobierno de evaluaciones de seguridad de funcionarios, obtener información para los Ministerios de Defensa y Relaciones Exteriores relativa a naciones extranjeras o a personas que no sean canadienses o residentes permanentes, dentro del territorio de Canadá - que

12. El Servicio obtendrá, por investigación o de otro modo, en la medida en que sea estrictamente necesaria, y analizará y retendrá, información e inteligencia relativa a actividades que razonablemente pueden ser sospechadas como constitutivas de amenazas a la seguridad del Canadá y, en relación a ello, informará y asesorará al Gobierno de Canadá.

La ley definió, además, en la sección 1 Interpretación, qué son amenazas a la seguridad de Canadá:

19 Sobre el control de la actividad de inteligencia en Estados Unidos de América, remitimos a, de UGARTE, José Manuel. Legislación de inteligencia: especialización y control, legitimidad y eficacia. Buenos Aires: Dunken, 1999; BUENOS AIRES. WOLA-SEDEM, 2000. Disponible en: www.sedem.org.gt. Acceso en: 2-1-2012; y UGARTE, José Manuel. El control público de la actividad de inteligencia en América Latina. Buenos Aires: Ediciones CICCUS, 2012.

20 Sobre el control de la actividad de inteligencia en Canadá versea UGARTE, José Manuel, obras citadas precedentemente. 
'Amenazas a la seguridad del Canadá' significa:

Espionaje o sabotaje contra Canadá, o en perjuicio a los intereses del Canadá, y que es clandestino o engañoso o involucra amenazas a alguna persona;

actividades influenciadas por países extranjeros dentro o en relación al Canadá que sean perjudiciales a los intereses del Canadá y que seann clandestinas o engañosas o involucran una amenaza para cualquier persona;

actividades dentro o relativas a al Canadá dirigidas hacia o en apoyo de la amenaza o uso de actos de seria violencia contra personas o bienes para el propósito de alcanzar un objetivo político dentro de Canadá o un estado extranjero, y

actividades dirigidas hacia el debilitamiento por actos ilegales encubiertos, o dirigidos hacia o concebidos finalmente para llevar a la destrucción o derrocamiento por violencia del sistema de gobierno constitucionalmente establecido de Canadá.

Pero no incluye la defensa de una causa, la protesta o el disenso legales, a menos que sean llevados a cabo conjuntamente con cualquiera de las actividades referidas en los párrafos a) a d).

Salta a la vista la claridad de la definición, que incluye actividades concretas que incluyen tanto contrainteligencia - espionaje, sabotaje, operaciones encubiertas de otros países en Canadá - como terrorismo, o incluso acciones dirigidas a derribar el sistema democrático por medios violentos. Pero además de la precisa determinación de los ámbitos de acción, se establece expresamente que serán objeto de las actividades de inteligencia y contrainteligencia del organismo en cuestión, las actividades y no el pensamiento o la defensa pacífica de un modo determinado de pensar.

Esta definición no brinda facultades para la producción de inteligencia sobre actividades lícitas ni sobre la política coyuntural, concentrando los esfuerzos de la inteligencia civil canadiense sobre las amenazas a la seguridad del Estado y del sistema democrático en Canadá.

\section{Italia}

En Italia, la Ley 124-2007 del 3 de agosto de 2007 sobre el Sistema de Informaciones para la seguridad de la República y nueva disciplina del Secreto, estableció un sistema de inteligencia caracterizado - en lo fundamental, y en breves líneas, por poseer un organismo de inteligencia exterior, la Agencia para la Información y la Seguridad Externa, un organismo de inteligencia interior, la Agencia para la Información y la Seguridad Interna, ambos bajo la dependencia directa del Primer Ministro, asistido por un órgano en el cual puede delegar parte de sus facultades, la Autoridad Delegada, y por un órgano de coordinación, elaboración de inteligencia estratégica 
y control, el Departamento de Información para la Seguridad, contando además con un consejo interministerial y un órgano de inteligencia militar con facultades limitadas, el Departamento de Informaciones y Seguridad del Estado Mayor de la Defensa.

En el caso italiano, cabe referir que por una parte, el artículo 6 estableció las normas relativas a la Agencia de Información y la Seguridad Externa (AISE) a la cual se le asigna la

competencia relativa a investigar y elaborar en el sector de su competencia todas las informaciones útiles para la defensa de la independencia, de la integridad y la seguridad de la República, así como en aplicación de acuerdos internacionales, ante amenazas provenientes del exterior, (...) la actividad en materia de contraproliferación correspondiente a los materiales estratégicos, así como la actividad de información para la seguridad, que tienen lugar fuera del territorio nacional, para proteger los intereses político, militar, económico, científico e industrial de Italia (...) y (...) detectar y luchar fuera del territorio nacional, contra la actividad de espionaje dirigida contra Italia y las actividades encaminadas a perjudicar los intereses nacionales.

\section{Por su parte, el artículo 7 creó la}

Agencia para la Información y la Seguridad Interna (AISI), a la cual es asignada la competencia relativa a investigar y elaborar en su sector de competencia toda la información útil para defender, así como en aplicación de acuerdos internacionales, la seguridad interna de la República y las instituciones democráticas fundadas en la Constitución ante toda amenaza, toda actividad subversiva y toda forma de agresión criminal y terrorista.

Corresponde también a la AISI la actividad de información para la seguridad, que tiene lugar en el interior del territorio nacional, para la protección de los intereses políticos, económicos, científicos e industriales de Italia.

Es también competencia de la AISI "detectar y contrarrestar en el interior del territorio nacional, la actividad de espionaje dirigida contra Italia y la actividad dirigida a dañar los intereses nacionales".

Aunque estas definiciones carecen de la importante precisión de la analizada anteriormente, resulta evidente que la actividad de inteligencia en el interior de Italia está orientada claramente a las amenazas contra la seguridad del Estado y del sistema democrático, hallándose excluida de su competencia la actividad política y asociativa legal.

\section{Reino Unido de Gran Bretaña e Irlanda del Norte}

A muy grandes rasgos, el sistema de inteligencia del Reino Unido comprende, bajo la autoridad superior del Primer Ministro, de cuyo gabinete dependen diversos órganos de coordinación, control interno, y producción 
de inteligencia estratégica, y bajo la dependencia directa del Secretario de Estado (ministro) cuyas competencias corresponden a las competencias del organismo, a) un organismo de inteligencia exterior, el Servicio Secreto de Inteligencia, b) un organismo de inteligencia interior y contrainteligencia, el Servicio de Seguridad, c) un organismo de inteligencia de señales, Cuarteles Generales de Comunicaciones, y d) un organismo de inteligencia de defensa y militar, Inteligencia de Defensa.

En materia de competencias de estos organismos, cabe destacar que la Ley sobre el Servicio de Seguridad de 1989 estableció que la función de dicho organismo estaría constituida por

la protección de la seguridad nacional y, en particular, su protección contra amenazas derivadas de espionaje, terrorismo y sabotaje, de las actividades de agentes de poderes extranjeros y de acciones dirigidas a derribar o debilitar la democracia parlamentaria por medios políticos, industriales o violentos (...) así como también (...) salvaguardar el bienestar económico del Reino Unido ante amenazas representadas por las acciones o intenciones de personas fuera de las Islas Británicas (sección 1).

La sección 2 incluyó también la estipulación relativa a que constituía responsabilidad del Director asegurar

que haya arreglos parar asegurar que no sea obtenida información por el Servicio sino en la medida en que sea necesario para el adecuado cumplimiento de sus funciones y no revelada por él excepto en la medida en que sea necesario para tal propósito o para el propósito de prevenir o detectar serios delitos; y que (...) el Servicio no tome ninguna acción para promover los intereses de cualquier partido político.

Cabe recordar asimismo, la Ley de los Servicios de Inteligencia de 1994, que dio carácter estatutario a los ya referidos Servicio Secreto de Inteligencia, y Cuartel General de Comunicaciones.

La sección I (1) de la ley referida dispuso respecto del primero, que sus funciones consistirían en

a) obtener y proveer información relativa a las acciones e intenciones de personas fuera de las Islas Británicas; y b) cumplir otras tareas relativas a las acciones e intenciones de tales personas (...) funciones que sólo serían ejercitables en los intereses de la seguridad nacional, con referencia particular a la defensa y políticas externas del Gobierno de su Majestad en el Reino Unido; o en el interés del bienestar económico del Reino Unido; o en apoyo de la prevención o detección de serios delitos.

También se impuso al Jefe del aludido organismo de inteligencia la obligación de garantizar 
que haya arreglos para asegurar que no sea obtenida información por ese Servicio de Inteligencia sino en la medida en que sea necesario para el adecuado desempeño de sus funciones y que ninguna información sea revelada por él excepto en la extensión en que sea necesario: para ese propósito; en interés de la seguridad nacional; para el propósito de la prevención o detección de serios delitos; para el propósito de cualesquiera procedimientos criminales, y que el Servicio de Inteligencia no tome cualquier acción para favorecer los intereses de cualquier partido político inglés.

De un modo muy similar, respecto del tercero de los organismos de inteligencia regulados legalmente, Cuarteles Generales de Comunicaciones, se estableció que su función consistiría en

monitorear e interferir emisiones electromagnéticas, acústicas y otras emisiones y cualquier equipo que produzca tales emisiones y obtener y proveer información derivada o relativa a tales emisiones o equipamiento y de material encriptado; y proveer asistencia y asesoramiento sobre-lenguajes, incluyendo terminología usada para asuntos técnicos, y criptografía y otros asuntos relativos a la protección de información y otro material, a las fuerzas armadas de la Corona, al Gobierno de Su Majestad en el Reino Unido o a un Departamento de Irlanda del Norte o a cualquier otra organización que sea determinada para los propósitos de esa sección en tal manera como sea especificada por el Primer Ministro.

Se estableció nuevamente que tales funciones serían ejercitables sólo

en los intereses de la seguridad nacional, con particular referencia a la defensa y políticas externas del Gobierno de Su Majestad en el Reino Unido; o en los intereses del bienestar económico del Reino Unido en relación a las acciones o intenciones de personas fuera de las Islas Británicas; o en apoyo de la prevención o detección de serios delitos.

Se dispuso, de modo similar a los casos anteriores, que

haya arreglos para asegurar que no sea obtenida información por el Servicio sino en la medida en que sea necesario para el adecuado cumplimiento de sus funciones y no revelada por él excepto en la medida en que sea necesario para tal propósito o para el propósito de prevenir o detectar serios delitos; y que (...) el Servicio no tome ninguna acción para promover los intereses de cualquier partido político.

Como puede advertirse, y aún cuando no puede advertirse una precisión comparable a la del caso canadiense, nuevamente podemos advertir que la actividad de inteligencia está orientada, en el caso del organismo de inteligencia exterior y en el de inteligencia de señales, a la política externa y a la defensa del país.

Respecto de la función relativa al interés del bienestar económico del Reino Unido - que podría constituir una potencial fuente de intromisión en 
actividades lícitas de los ciudadanos - las normas la limitan a las acciones e intenciones de personas fuera de las Islas Británicas, excepción hecha del Servicio Secreto de Inteligencia, cuyas funciones generales, no obstante, están también limitadas a las acciones e intenciones de personas fuera de las Islas Británicas, con lo que no existe el riesgo potencial señalado.

La distinción entre inteligencia exterior e interior permite mayor amplitud hacia el exterior, mientras que la delimitación es más rigurosa en el interior del país, particularmente en el caso del Servicio de Seguridad, dirigido en lo fundamental, a amenazas a la seguridad del Estado y del sistema democrático.

\section{Reino de Holanda}

La Ley de los Servicios de Inteligencia y Seguridad del 2002 estableció las funciones correspondientes a los dos principales organismos de inteligencia de Holanda: el Servicio General de Inteligencia y Seguridad, y el Servicio de Inteligencia y Seguridad de Defensa, dependientes respectivamente del Ministro del Interior y de Relaciones del Reino y del Ministro de Defensa, contando con un Coordinador.

Conforme previó el artículo 6, en el interés de la seguridad nacional, el Servicio General de Inteligencia y seguridad tiene las siguientes tareas:

a. Llevar a cabo investigaciones relativas a organizaciones que, y personas quienes, en razón de los objetivos que persiguen, o a través de sus actividades dan causa a serias sospechas de que constituyen un riesgo para la existencia continuada del sistema democrático legal, o para la seguridad u otros intereses vitales para el Estado; b. Llevan a cabo investigaciones de confiabilidad en seguridad como es referido en la Ley de Investigaciones para la Seguridad; c. promueven medidas para la protección de los intereses referidos en el apartado a, incluyendo medidas para la protección de la información que debe permanecer secreta por razones de seguridad nacional, e información perteneciente a aquellas partes del servicio público y la comunidad de negocios que, en opinión de los Ministros relevantes (de quienes dependen los organismos de inteligencia) son de vital importancia para la continuada existencia del orden social; d. conducir investigaciones relativas a otros países con respecto a asuntos designados por el Primer Ministro, Ministro de Relaciones Exteriores, de acuerdo con los ministros relevantes.

Por otra parte, el artículo 7 establece respecto al Servicio de Inteligencia y Seguridad de Defensa que

en el interés de la seguridad nacional (...) tiene las tareas de (...) llevar a cabo investigaciones: Sobre el potencial y las fuerzas armadas de otros países, a fin de alcanzar una balanceada composición y un uso efectivo de nuestras fuerzas armadas y; Sobre factores que son o que pueden ser de influencia en mantener y promover el sistema legal internacional, en todo cuanto las fuerzas armadas 
están o pueden llegar a estar involucradas; Llevar a cabo investigaciones de confiabilidad en seguridad, conforme está establecido en la Ley de Investigaciones de Seguridad; Llevar a cabo investigaciones para tomar medidas: para prevenir actividades dirigidas a perjudicar la seguridad o alistamiento de las fuerzas armadas; para promover una apropiada organización de la movilización y concentración de las fuerzas armadas; para promover una ágil preparación y despliegue de las fuerzas armadas...y promover medidas para proteger informaciones concernientes a las fuerzas armadas de cuyo secreto se requiere, y llevar a cabo investigaciones relativas a otros países, relativas a asuntos de relevancia militar que hayan sido encomendadas por el Primer Ministro, Ministro de Relaciones Exteriores, de acuerdo con los Ministros Relevantes.

Aquí nos encontramos con un diseño diverso: un organismo de inteligencia dedicado fundamentalmente a la contrainteligencia y a la inteligencia interior, en este último aspecto con relación a amenazas contra la seguridad del Estado y del sistema democrático, con facultades limitadas de inteligencia externa, desarrolladas a pedido del Primer Ministro; y un organismo de inteligencia de defensa, facultado para producir inteligencia de defensa e inteligencia estratégica militar. Ambos están facultados para llevar investigaciones de confiabilidad para la seguridad, evidentemente respecto de su propio personal y de los funcionarios de las áreas del Estado de las cuales dependen.

Sin advertir una precisión comparable a la del caso citado en primer término, se advierte que la actividad de inteligencia posee en este caso claros límites.

\section{Actividad de inteligencia ilimitada, y actividad de inteligencia con límites pobremente definidos: algunos ejemplos}

\section{Los criterios de determinación de competencias en Latinoamérica}

En materia de características de los organismos de inteligencia latinoamericanos, habremos de señalar en primer lugar la tendencia casi unánime de la actividad de inteligencia latinoamericana hacia la acumulación de facultades en un mismo organismo de inteligencia civil, en materia de inteligencia interior, exterior y contrainteligencia, sin efectuar diferenciación alguna en materia de inteligencia entre el interior y el exterior del país, o entre ciudadanos y residentes permanentes, y extranjeros.

En ningún caso se efectúa en Latinoamérica esta distinción, presente en la gran mayoría de los países que hemos señalado como dotados de significativo desarrollo institucional en la materia que nos ocupa.

La aludida tendencia, derivada en definitiva de las características que tuvieron los primeros organismos de inteligencia civil latinoamericanos que se constituyeron durante el conflicto Este - Oeste - o aún anteriores, como la SIDE argentina - se complementa también con la adopción por parte de 
diversos organismos de inteligencia de la región del esquema de la agencia central, que preconizado por Sherman Kent, doctrinario fundamental de la inteligencia estratégica estadounidense ${ }^{21}$, dio lugar a la creación, en la Ley de Seguridad Nacional de 1947, de la Agencia Central de Inteligencia (CIA), paradigmático organismo de inteligencia que inspiró en diversa pero importante medida a sus pares latinoamericanos..$^{22}$

Otra característica de los organismos de inteligencia de Latinoamérica es la amplitud e indeterminación de sus competencias, particularmente en el ámbito interno de sus países.

\section{Argentina}

En Argentina, la Ley No 25.520 de Inteligencia Nacional incluyó en su artículo $2^{\circ}$ definiciones básicas destinadas a determinar el ámbito normativo de la actividad de inteligencia, estableciendo que a los fines de la ley y de las actividades por ella reguladas, cabía entender, en primer lugar, como Inteligencia Nacional "a la actividad consistente en la obtención, reunión, sistematización y análisis de la información específica referida a los hechos, amenazas, riesgos y conflictos que afecten la seguridad exterior e interior de la Nación".

Si bien el requisito de que los hechos, amenazas, riesgos y conflictos afecten la seguridad exterior e interior de la Nación tiende a establecer un ámbito determinado, basta comparar esta definición con las contenidas en el capítulo precedente, para advertir que nos encontramos ante una amplitud mucho mayor que las contenidas en el capítulo precedente.

La circunstancia de no efectuarse distinción alguna entre los ámbitos externo e interno determina que mientras que para el ámbito interno el concepto aparece como excesivamente amplio, para el externo aparece por el contrario restringido, impidiendo - al menos teóricamente - escudriñar las oportunidades para la política exterior del país.

Respecto a la contrainteligencia, definida también en el referido artículo, como "la actividad propia del campo de la inteligencia que se realiza con el propósito de evitar actividades de inteligencia de actores que representen amenazas o riesgos para la seguridad del Estado Nacional", cabe observar que la expresión actores comprende desde Estados extranjeros hasta, hipotéticamente, personas, otorgando al término una amplitud muy superior a su concepción tradicional, comprensiva del contraespionaje, contrasabotaje,

21 KENT, Sherman. Inteligencia estratégica para la política mundial norteamericana.

22 Hemos analizado y discutido las características - y, a nuestro juicio, debilidades - de la concepción de la agencia central, sus efectos de aislamiento entre la inteligencia y la política, y las peculiaridades de su adopción por parte de los países latinoamericanos, en nuestro trabajo; UGARTE, José Manuel. La relación entre la inteligencia y la política y sus consecuencias y normas en las estructuras y normas de los Sistemas de Inteligencia. In: Actas de lo Seminario la Actividad de Inteligencia y los Desafíos Contemporáneos, diciembre de 2005, Agencia Brasileña De Inteligencia (ABIN), Congreso Nacional de la República Federativa de Brasil, Brasilia. Disponible en: www.fas. org. 
operaciones encubiertas por países extranjeros, y terrorismo internacional (en algunos ordenamientos).

También se definió en el mismo artículo a la Inteligencia Criminal como

la parte de la Inteligencia referida a las actividades criminales específicas que, por su naturaleza, magnitud, consecuencias previsibles, peligrosidad o modalidades, afecten la libertad, la vida, el patrimonio de los habitantes, sus derechos y garantías y las instituciones del sistema representativo, republicano y federal que establece la Constitución Nacional.

Cabe aquí advertir que pese a constituir la inteligencia criminal estrictamente hablando, parte de la actividad de seguridad pública, se la incluyó como parte de la actividad de inteligencia en sentido estricto. Cabe así destacar que mientras en Estados Unidos se asignó competencia a la FBI para cumplir tanto funciones de investigación criminal e inteligencia criminal, como de seguridad interna, contrainteligencia e inteligencia externa - predominantemente en territorio estadounidense - se distinguió claramente entre ambas funciones, bien que procurando, hasta el límite de lo posible, uniformar los procedimientos a emplear en uno y otro caso, en Argentina simplemente se asimiló la inteligencia criminal o inteligencia sobre el delito a la actividad de inteligencia. Así, se incluyó al órgano fundamental de producción de inteligencia criminal, y de dirección funcional de la actividad de los órganos de información e inteligencia de las policías y fuerzas de seguridad federales, la Dirección Nacional de Inteligencia Criminal, como miembro pleno del Sistema de Inteligencia Nacional, sujetándolo a las mismas normas que a los restantes organismos de inteligencia que previó, la Secretaría de Inteligencia - organismo de inteligencia exterior, interior y contrainteligencia de muy amplias facultades, dependiente directamente del Presidente de la Nación y cuyas facultades incluyen la de participar a requerimiento del Consejo de Seguridad Interior en la producción de inteligencia criminal.

No obstante, la ley definió muy estrictamente a la Inteligencia Estratégica Militar, también en el artículo en cuestión, "como la parte de la Inteligencia referida al conocimiento de las capacidades y debilidades del potencial militar de los países que interesen desde el punto de vista de la defensa nacional, así como el ambiente geográfico de las áreas estratégicas operacionales determinadas por el planeamiento estratégico militar", estableciendo, además (artículo 10 último párrafo) que: "Los organismos de inteligencia de las Fuerzas Armadas tendrán a su cargo la producción de la inteligencia estratégica operacional y la inteligencia táctica necesarias para el planeamiento y conducción de operaciones militares y de la inteligencia técnica específica". Se trata, como puede verse, una concepción estricta de la inteligencia militar, no frecuente en Latinoamérica.

También cabe destacar que pese a la amplitud de su concepto de inteligencia y a la virtual confusión entre seguridad pública e inteligencia que 
campea en ella, la Ley № 25520 previó límites a la actividad de inteligencia. Ellos pueden ser hallados en el artículo $4^{\circ}$, que estableció que:

\begin{abstract}
Ningún organismo de inteligencia podrá: 1. Realizar tareas represivas, poseer facultades compulsivas, cumplir, por sí, funciones policiales ni de investigación criminal, salvo ante requerimiento específico realizado por autoridad judicial competente en el marco de una causa concreta sometida a su jurisdicción, o que se encuentre, para ello, autorizado por ley. 2. Obtener información, producir inteligencia o almacenar datos sobre personas, por el solo hecho de su raza, fe religiosa, acciones privadas, u opinión política, o de adhesión o pertenencia a organizaciones partidarias, sociales, sindicales, comunitarias, cooperativas, asistenciales, culturales o laborales, así como por la actividad lícita que desarrollen en cualquier esfera de acción. 3. Influir de cualquier modo en la situación institucional, política, militar, policial, social y económica del país, en su política exterior, en la vida interna de los partidos políticos legalmente constituidos, en la opinión pública, en personas, en medios de difusión o en asociaciones o agrupaciones legales de cualquier tipo. 4. Revelar o divulgar cualquier tipo de información adquirida en ejercicio de sus funciones relativa a cualquier habitante o a personas jurídicas, ya sean públicas o privadas, salvo que mediante orden o dispensa judicial.
\end{abstract}

Aunque tales límites no son por cierto infranqueables, máxime en un sistema que a las más que importantes facultades de la Secretaría de Inteligencia, une la total inexistencia de controles de la actividad de inteligencia en el seno del órgano ejecutivo. ${ }^{23}$

\title{
República Federativa de Brasil
}

En Brasil, la Ley No 9.883: Que instituye el Sistema Brasileño de Inteligencia y Crea la Agencia Brasileña de Inteligencia (...) definió a la inteligencia (artículo $1^{\circ}$, apartado 2) como "la actividad que tiene por objeto la obtención, análisis y diseminación de conocimientos dentro y fuera del territorio nacional sobre hechos o situaciones de inmediata o potencial influencia sobre el proceso decisorio y la acción gubernamental y sobre la salvaguardia y la seguridad de la sociedad y del Estad". A su vez, el artículo 2 apartado 2 de la referida ley estableció que el "Sistema Brasileño de Inteligencia (...) es responsable por el proceso de obtención, análisis y diseminación de la información necesaria al proceso decisorio del Poder Ejecutivo, así como por la salvaguardia de la información contra el acceso de personas o de órganos no autorizados". Asimismo, el artículo $4^{\circ}$ apartado 1 incluye entre las facultades de la "Agencia Brasileña de Inteligencia ABIN - agencia central - las de planear y ejecutar acciones, inclusive sigilosas, relativas a la obten-

23 Verse sobre estos aspectos, de José Manuel Ugarte: El control público de la actividad de inteligencia en América Latina. 
ción y análisis de datos para la producción de conocimientos destinados a asesorar al Presidente de la República".

Como puede advertirse, en Brasil la actividad de inteligencia, incluyendo aquellas acciones que suponen la obtención de información por medios encubiertos o de fuentes no públicas, es concebida como un aspecto ordinario de la acción gubernamental corriente, con el único límite del need to know del Presidente de la República. No existe límite alguno al accionar de la inteligencia.

En cambio, la contrainteligencia es definida como "la actividad que tiene por objeto neutralizar la inteligencia adversa" concepto que aunque no exento de vaguedad, es más cercano al concepto clásico de contrainteligencia, que la definición examinada en el caso anterior.

La amplitud del ámbito conceptual de la inteligencia que caracteriza al Sistema Brasileño de Inteligencia (SISBIN) se evidencia al disponer el artículo $2^{\circ}$ de la precedentemente referida ley, que:

Los órganos y entidades de la Administración Pública Federal que, directa o indirectamente, puedan producir conocimientos de interés para las actividades de inteligencia, en especial aquellos responsables por la defensa externa, seguridad interna y relaciones exteriores, constituirán el Sistema Brasileño de Inteligencia, a través de un acto del Presidente de la República.

Ratifica la amplitud mencionada la disposición contenida en el artículo referido en cuanto a la misión del SISBIN, al disponerse que aquél "es responsable por el proceso de obtención, análisis y diseminación de la información necesaria al proceso decisorio del Poder Ejecutivo, así como por la salvaguardia contra el acceso a personas u órganos no autorizados". La ley faculta además, en el mismo artículo, a que mediante "ajustes específicos y convenios, oído el órgano de control externo competente de la actividad de inteligencia, las Unidades de la Federación" puedan integrar también el SISBIN.

Las normas relativas a las facultades de la agencia central del SISBIN se caracterizan por una amplitud similar.

Así, el artículo $4^{\circ}$ de dicha ley atribuyó a la $A B I N$ facultades para "planear y ejecutar acciones, inclusive sigilosas, relativas a la obtención y al análisis de datos para la producción de conocimientos destinados a asesorar al Presidente de la República" reiterando la ya expresada amplitud; todo lo que el Presidente desee conocer - en cualquier ámbito - puede determinar la realización de acciones sigilosas para obtenerlo.

Ello, sin perjuicio de destacar que al disponer el Decreto $N^{\circ} 4376 / 2002$ y su modificatorio № 4872/2003 que fuera el Gabinete de Seguridad Institucional de la Presidencia de la República, dirigido por su ministro-jefe - del cual ha pasado a depender la ABIN - el órgano de coordinación de las actividades de inteligencia federal, la ABIN vio transferidas parte de las facultades 
de coordinación y supervisión que poseía respecto de las actividades de inteligencia en Brasil que le confiaba el artículo $3^{\circ}$ de la ley en análisis, en beneficio del referido Gabinete de Seguridad Institucional.

Para evaluar la amplitud del concepto de inteligencia antes señalado, es útil considerar que conforme al ya referido Decreto № 4376/2002 modificado, forman parte del SISBIN, además de los organismos de inteligencia militares y de los órganos de inteligencia de las instituciones federales de seguridad, el Ministerio de Hacienda por intermedio de la Secretaría Ejecutiva del Consejo de Control de Actividades Financieras de la Secretaría de Ingresos Federales y del Banco de Brasil, el Ministerio de Trabajo y Empleo por intermedio de la Secretaría Ejecutiva, el Ministerio de Salud por intermedio del Gabinete del Ministro de Estado y de la Agencia de Vigilancia Sanitaria (ANVISA), el Ministerio de Acción Social por intermedio de la Secretaría Ejecutiva, el Ministerio de Ciencia y Tecnología por intermedio del Gabinete del Ministro de Estado, el Ministerio de Integración Nacional por intermedio de la Secretaría Nacional de Defensa Civil. En definitiva, evidente resulta que el concepto de inteligencia aplicado en Brasil excede con mucho los ámbitos de las relaciones internacionales, la defensa y la seguridad.

También cabe destacar la creación, por Decreto No 3448/2000, modificado por su similar N ${ }^{\circ} 3695 / 2000$, dentro del ámbito del Sistema Brasileño de Inteligencia, del Subsistema Brasileño de Seguridad Pública, con la finalidad de "coordinar e integrar las actividades de inteligencia de seguridad pública en todo el país, así como suministrar a los gobiernos federal, estaduales y municipales, informaciones que apoyen la toma de decisiones en ese campo (artículo 10)".

Estableció asimismo el Artículo $2^{\circ}$ que: "Integran el Subsistema de Inteligencia de Seguridad Pública los Ministerios de Justicia, de Hacienda, de Defensa, y de Integración Nacional y el Gabinete de Seguridad Institucional de la Presidencia de la República".

No obstante, el apartado $2^{\circ}$ de este artículo facultó también a integrar el Subsistema, a los órganos de inteligencia de seguridad pública del Distrito Federal y de los Estados.

Se asignó el carácter de órgano central del Subsistema (artículo $2^{\circ}$, apartado 1) a la Secretaría Nacional de Seguridad Pública del Ministerio de Justicia (inicialmente había sido otorgado tal carácter a la ABIN).

Dispuso su apartado 3, que: "Cabe a los integrantes del Subsistema, en el ámbito de sus competencias, identificar, acompañar y evaluar amenazas reales o potenciales de seguridad pública, y producir conocimientos e informaciones que apoyen acciones para neutralizar, cohibir y reprimir actos criminales de cualquier naturaleza".

El artículo $3^{\circ}$ estableció la integración del 
Consejo Especial del Subsistema de Seguridad Pública,(...) órgano de deliberación colectiva, con la finalidad de establecer normas para las actividades de inteligencia de seguridad pública, que tendrá la siguiente composición:

I - como miembros permanentes, con derecho a voto; a) el Secretario Nacional de Seguridad Pública, que lo presidirá; b) un representante del órgano de inteligencia del Departamento de Policía Federal y otro del área operacional; c) dos representantes del Ministerio de Hacienda, siendo uno de ellos del Consejo de Control de Actividades Financieras (COAF) y otro de la Coordinación General de Búsqueda e Investigación (COPEI) de la Secretaría de Ingresos Federales; d) dos representantes del Ministerio de Defensa; e) un representante del Gabinete de Seguridad Institucional de la Presidencia de la República; f) un representante de la Defensa Civil del Ministerio de Integración Nacional; y g) un representante de la Agencia Brasileña de Inteligencia.

II. como miembros eventuales, sin derecho a voto, un representante de cada uno de los órganos de que trata el apartado $2^{\circ}$ del artículo $2^{\circ}$ (...) (órganos de inteligencia de seguridad pública del Distrito Federal y de los Estados).

Cabe pues, advertir que en Brasil - como sucede también hoy en Argentina - existe una identificación total entre los sistemas de seguridad pública y de inteligencia. Aquí no se trata, como sucede en Estados Unidos de América, de la creación de vínculos que permiten la transmisión por parte del sistema de seguridad pública al sistema de inteligencia, preservando los derechos individuales, o incluso la asignación a un mismo organismo - la $\mathrm{FBI}$ - de funciones correspondientes a ambos ámbitos, procurando evitar los riesgos institucionales ínsitos en dicha circunstancia, con controles. En Brasil y en Argentina, y, como veremos, en el resto de Latinoamérica que cuenta con legislación orgánica de inteligencia y con controles - y con mayor razón, en los restantes - la llamada inteligencia policial integra plenamente los respectivos sistemas de inteligencia, con la diferencia en el caso de Brasil que el órgano central del Subsistema de Inteligencia no es la ABIN, organismo de inteligencia civil fundamental, sino la Secretaría de Seguridad Pública. En cambio, en Argentina, el órgano central es, para todos los casos, la Secretaría de Inteligencia.

\section{Perú}

La Ley No 28.664 Ley del Sistema de Inteligencia Nacional SINA y de la Dirección Nacional de Inteligencia DINI (2006), superando la amplitud del concepto de inteligencia y de las competencias de los órganos y organismos que lo integraban que tenía su predecesora Ley No 27.479, incluyó en su artículo $3^{\circ}$, relativo al Objetivo de la Actividad de Inteligencia, la mención relativa a que dicho objetivo consistía en

proporcionar oportunamente a través del Órgano Rector del Sistema de Inteligencia Nacional SINA, al Presidente Constitucional de la República y al Consejo 
de Ministros, el conocimiento útil, obtenido mediante el procesamiento de las informaciones, sobre las amenazas y riesgos actuales y potenciales, que puedan afectar la seguridad nacional y el ordenamiento constitucional de la República.

La referencia a las amenazas y riesgos brinda cierto grado de concreción al concepto. Por otra parte, respecto a la estipulación relativa a que aquéllos deben afectar la seguridad nacional y el ordenamiento constitucional, la ley incluyó en un Glosario de Términos - previsión siempre útil - una definición de seguridad nacional, como: "Condición de viabilidad, estabilidad, continuidad y bienestar del Estado y la Nación, a través de políticas públicas especializadas sectoriales de defensa y orden interno, inteligencia y en campos o dominios no militares; para protegerlo y ponerlo fuera de peligro, ante situaciones de amenaza, daño potencial o riesgo". Esta definición, si bien no tiene el carácter estricto de los vigentes en otras latitudes, mantiene de todos modos cierta especificidad; pudiéndose advertir que la referencia a situaciones de amenaza, daño potencia o riesgo contribuye a evitar el empleo indiscriminado de la actividad de inteligencia como auxiliar de la política gubernamental ordinaria, aún dentro del país y respecto de los propios ciudadanos.

También cabe referir como aspecto a nuestro juicio positivo lo establecido en el artículo $4^{\circ}$ de la Ley $N^{\circ} 28.664$ con relación a los principios de la actividad de inteligencia, en los cuales se destacan cuatro principios que consideramos fundamentales para una adecuada y eficaz actividad de inteligencia: "legalidad, legitimidad, control democrático, pertinencia, circulación restringida, especialidad y planificación".

Un aspecto de interés, no frecuente en Latinoamérica, es el que surge del artículo $2^{\circ}$ Alcances y Límites, que prescribe que: "La presente Ley desarrolla los alcances y establece los límites que deben observar los organismos del Sistema de Inteligencia Nacional - SINA - y los que señale la presente Ley". Particularmente, el reconocimiento de que la actividad de inteligencia posee límites, es de importancia para nuestro análisis.

Cabe destacar no obstante que la ley adhiere a la tendencia latinoamericana de no distinguir entre los ámbitos externo e interno del país, lo que lleva a asignar a la actividad de inteligencia un ámbito similar en uno y otro caso.

También resulta inevitable señalar - otra característica latinoamericana - la inclusión plena de la inteligencia policial dentro del ámbito conceptual y normativo de la ley, aplicándosele las mismas normas que para la inteligencia nacional o de Estado, inteligencia estratégica nacional y militar. Ello comprende no sólo a la Dirección General de Inteligencia del Ministerio del Interior, DIGIMIN, sino al órgano de inteligencia de la Policía Nacional de Perú, DIRIN.

En definitiva, la Ley № 28.664 representó un avance significativo respecto de su predecesora en relación a la existencia de límites y contenido 
preciso y concreto en materia de inteligencia interior. No obstante, también en Perú se produce la identificación o asimilación de la inteligencia policial a la actividad de inteligencia, tendiéndose a confundir.

\section{Chile}

En Chile, la Ley No 19.974 sobre el Sistema de Inteligencia del Estado, de creación de la Agencia Nacional de Inteligencia (ANI) definió en su artículo $2^{\circ}$, a los fines de la aplicación de la ley y de la regulación de las actividades normadas por ella, a la inteligencia como "el proceso sistemático de recolección, evaluación y análisis de información, cuya finalidad es producir conocimiento útil para la toma de decisiones" definiendo también a la contrainteligencia como "aquella parte de la actividad de inteligencia cuya finalidad es detectar, localizar y neutralizar las acciones de inteligencia desarrolladas por otros Estados o por personas, organizaciones o grupos extranjeros, o por sus agentes locales, dirigidas contra la seguridad del Estado y la defensa nacional".

Salta a la vista la amplitud del concepto; encontramos nuevamente la inteligencia ilimitada, cuyo único límite es el need to know de cualquier funcionario, transformada en insumo de cualquier decisión gubernamental en cualquier ámbito o terreno.

También se caracteriza de manera similar el concepto de contrainteligencia: los grupos e inclusive las personas.

Tamaña amplitud, que bien puede comprender la inteligencia política, halla no obstante un límite importante en lo dispuesto en el artículo 23, con relación a los procedimientos especiales de obtención de información, cuya utilización halla una doble limitación: que la información a obtener por tales medios "sea estrictamente indispensable para el cumplimiento de los objetivos del Sistema y no pueda ser obtenida de fuentes abiertas" - en definitiva un principio de subsidiaridad - y, más concluyentemente, se establece que los aludidos procedimientos "estarán limitados exclusivamente a actividades de inteligencia y contrainteligencia que tengan por objetivo resguardar la seguridad nacional y proteger a Chile y su pueblo de las amenazas del terrorismo, el crimen organizado y el narcotráfico".

De ese modo, mientras la actividad de inteligencia en sí es virtualmente ilimitada, la utilización de procedimientos especiales de obtención de información - los llamados medios encubiertos, vulneratorios de la privacidad - tiene, en cambio, muy claros y precisos límites. Aunque creemos preferible que tales límites fueran los de la actividad de inteligencia en sí, parece evidente que del modo indicado, los previsibles efectos negativos quedan menguados.

Sin perjuicio de ello, cabe destacar que Chile reproduce plenamente la ya señalada característica de integrar plenamente a la inteligencia policial 
dentro del Sistema de Inteligencia, bien que quepa observar que el referido Sistema tiene una coordinación con muy limitadas facultades ${ }^{24}$ por parte del organismo de inteligencia civil fundamental - la ANI - lo que determina que en el caso de la seguridad pública, sea el nuevo Ministerio del Interior y Seguridad Pública creado por la Ley № 20.502 (2011) quien, a través de Carabineros y de la Policía de Investigaciones, puede ejercer la coordinación al respecto, aunque la ley citada no le otorga facultades expresas al respecto.

\section{Estados Unidos Mexicanos}

En México, la Ley de Seguridad Nacional del 2005, basada en un concepto de seguridad nacional de significativa amplitud, contuvo también la primera regulación legislativa de la actividad de inteligencia en el país.

Un aspecto fundamental para determinar la amplitud y competencias de la actividad de inteligencia en México es la definición de Seguridad Nacional contenida en el artículo $3^{\circ}$ de la ley, que estableció que:

Para efectos de esta Ley, por Seguridad Nacional se entienden las acciones destinadas de manera inmediata y directa a mantener la integridad, estabilidad y permanencia del Estado Mexicano, que conlleven a: I. La protección de la nación mexicana frente a las amenazas y riesgos que enfrente nuestro país; II La preservación de la soberanía e independencia nacionales y la defensa del territorio; III. El mantenimiento del orden constitucional y el fortalecimiento de las instituciones democráticas de gobierno; IV. El mantenimiento de la unidad de las partes integrantes de la Federación señaladas en el artículo 43 de la Constitución Política de los Estados Unidos Mexicanos; V La defensa legítima del Estado Mexicano respecto de otros Estados o sujetos de derecho internacional, y VI La preservación de la democracia, fundada en el desarrollo económico social y político del país y sus habitantes.

Como puede advertirse, la "protección de la nación mexicana frente a las amenazas y riesgos" es sólo uno de los aspectos de la seguridad nacional, que comprende aspectos tan amplios como "el fortalecimiento de las instituciones democráticas de gobierno y la preservación de la democracia, fundada en el desarrollo económico social y político del país y sus habitantes". Por ello, la nutrida y variada nómina de amenazas prevista en el artículo $5^{\circ}$, constituye sólo una parte del aludido concepto de seguridad nacional.

Respecto a la inteligencia, ésta es definida en el artículo 29 como "el conocimiento obtenido a partir de la recolección, procesamiento, diseminación y explotación de información, para la toma de decisiones en materia de Seguridad Nacional", disponiéndose además, que la información sólo puede ser "recabada, compilada, procesada y diseminada con fines de

24 Remitimos al análisis efectuado en UGARTE, José Manuel. República de Chile. In: El control público de la actividad de inteligencia en América Latina, p.275 y siguientes. 
Seguridad Nacional por las instancias autorizadas", limitación no demasiado importante, dada la amplitud del aludido concepto.

México dispone de un organismo de inteligencia civil, el Centro de Investigación y Seguridad Nacional (CISEN) y de organismos de inteligencia de las Fuerzas Armadas, contando asimismo con órganos de inteligencia policial, entre los que se destaca el perteneciente a la Policía Federal, dependiente de la Secretaría Nacional de Seguridad Pública. La Ley de Seguridad Nacional no estableció coordinación alguna en materia de inteligencia, disponiendo en cambio en su artículo 31 que: "Al ejercer atribuciones propias de la producción de inteligencia, las instancias gozarán de autonomía técnica y podrán hacer uso de cualquier método de recolección de información, sin afectar en ningún caso las garantías individuales ni los derechos humanos". No obstante, la reglamentación atribuye al CISEN muy limitadas facultades de coordinación, como se verá.

Respecto al CISEN, el artículo 18 le otorgó atribuciones con amplitud correlativa a la ya examinada del concepto de seguridad nacional, entre las que se destacan las de:

I. Operar tareas de inteligencia como parte del sistema de seguridad nacional que contribuyan a preservar la integridad, estabilidad y permanencia del Estado Mexicano, a dar sustento a la gobernabilidad y a fortalecer el Estado de Derecho (...) y (...) V. Proponer medidas de prevención, disuasión, contención y desactivación de riesgos y amenazas que pretendan vulnerar el territorio, la soberanía, las instituciones nacionales, la gobernabilidad democrática o el Estado de Derecho.

Tal amplitud se incrementó significativamente en el Reglamento para la Coordinación de Acciones Ejecutivas en Materia de Seguridad Nacional (2006) al establecerse, entre las vertientes en base a las cuales serían atendidos los problemas relativos a la Seguridad Nacional, la vertiente de Inteligencia Estratégica (artículos 5 y 7 ), la cual debía orientarse por: "I. Seguridad: La generación de datos para detectar, prevenir, disuadir, contener y posibilitar la desactivación de Amenazas, y II. Desarrollo: La generación de datos para la ejecución de proyectos estratégicos para el desarrollo del Estado mexicano, de conformidad con los lineamientos que establezca el Consejo".

Como puede advertirse, difícilmente exista algún aspecto que no pueda considerarse comprendido en estas definiciones y quedar, por ende, no comprendido en la inteligencia estratégica. La inclusión del desarrollo dentro del ámbito conceptual de la seguridad - muy frecuente en Latinoamérica, a partir de las doctrinas de seguridad continental y fronteras ideológicas- acaba incluyendo dentro de dicho ámbito a la política ordinaria del Estado, con la consiguiente amplitud del ámbito abarcado por la actividad de inteligencia.

Cabe asimismo señalar que en el Reglamento, fueron asignadas al CISEN facultades de conducción de acciones para la integración de 
inteligencia estratégica (artículo 9, apartado II) y 28, pudiendo requerir a las instancias pertinentes la información con que contaren (artículo 28, $2^{\circ}$ do párrafo), estableciéndose, de ese modo, una coordinación limitada en materia de inteligencia, anteriormente ausente.

En definitiva, cabe señalar que la actividad de inteligencia en Estados Unidos Mexicanos tiene una amplitud muy significativa; difícilmente pueda concebirse un aspecto que no pueda estar comprendido por ella. Bajo el binomio seguridad-desarrollo, clásico de las doctrinas de seguridad continental y fronteras ideológicas latinoamericanas, puede abarcar virtualmente la totalidad de la política gubernamental. Respecto de la seguridad pública, regida fundamentalmente por la Ley General del Sistema Nacional de Seguridad Pública (2009) y por la Ley de la Policía Federal, incluye mecanismos de intercambio de información policial - materializados en la Plataforma México - previendo además la Ley de la Policía Federal (2009) la obtención de información relativa al delito por la nombrada institución y la consiguiente producción de inteligencia sobre el delito o inteligencia criminal.

Si bien no existe en este aspecto identificación entre los ámbitos de la seguridad pública y la inteligencia, hallándose prevista la actividad del CISEN, exclusivamente para la seguridad nacional, la amplitud del concepto de seguridad nacional y la ausencia de claros límites entre éste concepto y el de seguridad pública determinan en consecuencia que, como hemos señalado, no existan en la práctica límites concretos para la actividad de inteligencia en México.

\section{República de Guatemala}

En Guatemala, tras debates, marchas y contramarchas en materia de reforma de la actividad de inteligencia tras la firma de los Acuerdos de Paz, el Decreto Legislativo No 71-2005 (Ley de de la Dirección General de Inteligencia Civil - DIGICI) y especialmente la Ley Marco del Sistema Nacional de Seguridad (Decreto Legislativo 18-2008) dieron forma al Sistema de Inteligencia de Estado guatemalteco.

Cabe señalar que esta última ley, de modo similar a la Ley de Seguridad Nacional de Estados Unidos Mexicanos, pero con un grado muy superior de concreción y precisión, incluyó dentro del mismo texto legal los ámbitos de la Seguridad Exterior, la Seguridad Interior, la Inteligencia de Estado y la Gestión de Riesgos y Defensa Civil.

El ámbito de la Inteligencia de Estado fue definido en el artículo 17 como "la capacidad de articular en los ámbitos de la seguridad interior y seguridad exterior la información e inteligencia en grado de certeza frente a amenazas, riesgos y vulnerabilidades internas y externas". Si bien está lejano del grado de concreción advertido en los países cuyas normas en la 
materia hemos puesto como ejemplo, este concepto es razonablemente concreto, teniendo en cuenta el contexto latinoamericano.

Por otra parte, el artículo 23, referido al Sistema de Inteligencia de Estado, estableció que dicho Sistema

\begin{abstract}
es el conjunto de instituciones, procedimientos y normas que abordan con carácter preventivo, las amenazas y riesgos a la seguridad de la Nación, mediante la necesaria coordinación de las funciones de inteligencia estratégica, civil y militar; así como de cada una de ellas en su ámbito de actuación. Es responsable de producir inteligencia y conducir la contrainteligencia, trasladándola a las respectivas autoridades superiores, de conformidad con las atribuciones asignadas por la presente ley y demás disposiciones pertinentes.
\end{abstract}

El Sistema de Inteligencia de Estado lo coordina la Secretaría de Inteligencia de Estado.

Preciso es destacar que el párrafo final del artículo 25, relativo a funciones de la referida Secretaría, veda a ésta "realizar ni participar en investigaciones en beneficio de particulares, como también actuar en forma alguna para limitar o entorpecer el ejercicio de los derechos políticos y de emisión del pensamiento".

Cabe consignar que, en suma, el Sistema de Inteligencia de Estado posee competencias que si bien no están definidas con el grado de precisión que es dable advertir en los países utilizados como modelo, presentan en el contexto latinoamericano cierto grado de concreción, así como ciertos límites.

\title{
Repúlica del Ecuador
}

En Ecuador, la Ley de Seguridad Pública y del Estado que derogó la antigua Ley de Seguridad Nacional, que constituyera un reducto de las concepciones de seguridad nacional y fronteras ideológicas que imperaran en Latinoamérica durante el Conflicto Este-Oeste, sustituyó el antiguo concepto de seguridad nacional por otro concepto también de amplitud significativa, el de seguridad integral del Estado democrático de derechos y justicia, bien que privado de las connotaciones ideológicas de su predecesor. Por ello, el objeto de la ley, significativamente ambicioso, no era sino el de garantizar "el orden público, la convivencia, la paz y el buen vivir, en el marco de sus derechos y deberes como personas naturales y jurídicas, comunidades, pueblos, nacionalidades y colectivos, asegurando la defensa nacional, previniendo los riesgos y amenazas de todo orden, a través del Sistema de Seguridad Pública y del Estado".

En consecuencia, el artículo $2^{\circ}$ De los ámbitos de la ley dispuso que al amparo de aquélla 
se establecerán e implementarán políticas, planes, estrategias y acciones oportunas para garantizar la soberanía e integridad territorial, la seguridad de las personas, comunidades, pueblos, nacionalidades y colectivos, e instituciones, la convivencia ciudadana de una manera integral, multidimensional, permanente, la complementariedad entre lo público y lo privado, la iniciativa y aporte ciudadanos, y se establecerán estrategias de prevención para tiempos de crisis o grave conmoción social.

Se protegerá el patrimonio cultural, la diversidad biológica, los recursos genéticos, los recursos naturales, la calidad de vida ciudadana, la soberanía alimentaria; y en el ámbito de la seguridad del Estado, la protección y control de los riesgos tecnológicos y científicos, la tecnología e industria militar, el material bélico, tenencia y porte de armas, materiales, sustancias biológicas y radioactivas, etc.

\section{En definitiva, no poco cometido para una ley.}

Pese a la ya señalada amplitud, preciso es advertir la existencia de una clara delimitación entre los ámbitos de la defensa, comprendiendo la defensa de la soberanía y de la integridad territorial, y asignada a los Ministerios de Defensa, Relaciones Exteriores y a las Fuerzas Armadas, de orden público, asignado al Ministerio de Gobernación, Policía y Cultos y a la Policía Nacional, de la prevención y protección de la convivencia y seguridad ciudadanas, asignada a todos los órganos del país, y la gestión de riesgos, encomendada al organismo especializado del Sistema Nacional de Gestión de Riesgos.

La ley contiene las normas legales actualmente vigentes en Ecuador en materia de inteligencia, a la que definió en su artículo 14 como "la actividad consistente en la obtención, sistematización y análisis de la información específica referida a las amenazas, riesgos y conflictos que afecten la seguridad integral. La información de inteligencia es sustancial para la toma de decisiones en materia de seguridad".

Como puede advertirse, referido el concepto de inteligencia al concepto de seguridad integral, la significativa amplitud de dicho concepto se traslada al de seguridad. Pocos aspectos de la vida ecuatoriana quedan fuera de los alcances de la inteligencia.

Con cierta vaguedad, la contrainteligencia es definida como "la actividad de inteligencia que se realiza con el propósito de evitar o contrarrestar la efectividad de las operaciones de inteligencia que representan amenazas o riesgos para la seguridad".

Cabe señalar además que, como sucede en otros países latinoamericanos, Ecuador integra en su Sistema Nacional de Inteligencia a los órganos de información e inteligencia militares y policiales (artículo 15 inciso c), Ley de Seguridad Pública y del Estado) cuya actividades y funcionamiento es coordinada, articulada e integrada por la Secretaría Nacional de Inteligencia (SENAIN), nuevo organismo de inteligencia civil creado por Ecuador con anterioridad a la sanción de la ley antes referida, a través del Decreto No 1768/2009, y que une a las ya referidas funciones de coordinación, las 
de planificación y de producción de inteligencia, incluyendo inteligencia estratégica.

Cabe señalar, a mayor abundamiento, que en el Reglamento de la Ley de Seguridad Pública y del Estado (2010) se estableció específicamente (art.7) que integrarían el "Sistema Nacional de Inteligencia, a. La Secretaría Nacional de Inteligencia; b. Los Subsistemas de Inteligencia Militar; c. Los Subsistemas de Inteligencia Policial; d. La Unidad de Inteligencia Financiera; e. El Servicio de Protección Presidencial; y, f. Unidad de Gestión de Seguridad Interna de la Presidencia de la República".

Es decir, que en Ecuador ninguna distinción legal se efectúa entre la inteligencia policial, la inteligencia militar y la inteligencia estratégica, hallándose todas bajo el mismo régimen legal.

\section{República de Colombia}

En Colombia, la declaración por la Corte Constitucional de inconstitucionalidad de la Ley No 1288/2009 sobre normas destinadas a fortalecer el marco legal que permite a los organismos de inteligencia y contrainteligencia cumplir con su misión constitucional y legal, por razones de forma - no poseer el carácter de ley estatutaria, requerido por su naturaleza, dio lugar a la tramitación de un nuevo cuerpo normativo homónimo, que sí posee el carácter indicado, y que tras ser aprobado por el Senado y la Cámara de Representantes, aguarda su revisión por la Corte Constitucionalidad. El nuevo texto supera claramente al anterior en diversos aspectos - fundamentalmente, precisión en sus definiciones, límites y controles.

Iniciando una breve revista de su contenido, en relación al tema que nos ocupa, diremos que su artículo $2^{\circ}$ definió a la función de inteligencia y contrainteligencia como

aquélla que desarrollan los organismos especializados del Estado del orden
nacional, utilizando medios humanos o técnicos para la recolección, procesa-
miento, análisis y difusión de información, con el objetivo de proteger los derechos
humanos, prevenir y combatir amenazas internas o externas contra la vigencia del
régimen democrático, el régimen constitucional y legal, la seguridad y la defensa
nacional, y cumplir los demás fines enunciados en esta Ley.

Como puede advertirse, y más allá de señalar que no parece sencillo promover los derechos humanos a través del empleo de la actividad de inteligencia, resulta innegable el esfuerzo legislativo por definir un ámbito razonablemente limitado para las actividades de inteligencia y contrainteligencia.

Al mismo objetivo contribuye lo dispuesto en el artículo $4^{\circ}$ en relación a los Límites y fines de la función de inteligencia y contrainteligencia, donde se establece que tal función 
estará limitada en su ejercicio al respeto de los derechos humanos y al cumplimiento estricto de la Constitución, la Ley y el Derecho Internacional Humanitario y el Derecho Internacional de los Derechos Humanos. En especial, la función de inteligencia estará limitada por el principio de reserva legal que garantiza la protección de los derechos a la honra, al buen nombre, a la intimidad personal y familiar, y al debido proceso.

Ninguna información de inteligencia y contrainteligencia podrá ser obtenida con fines diferentes de:

Asegurar la consecución de los fines esenciales del Estado, la vigencia del régimen democrático, la integridad territorial, la soberanía, la seguridad y la defensa de la Nación;

Proteger las instituciones democráticas de la República, así como los derechos de las personas residentes en Colombia y de los ciudadanos colombianos en todo tiempo y lugar - en particular los derechos a la vida y la integridad personal - frente a amenazas tales como el terrorismo, el crimen organizado, el narcotráfico, el secuestro, el tráfico de armas, municiones, explosivos y otros materiales relacionados, el lavado de activos, y otras amenazas similares; y

Proteger los recursos naturales y los intereses económicos de la Nación.

En ningún caso la información de inteligencia y contrainteligencia será recolectada, procesada o diseminada por razones de género, raza, origen nacional o familiar, lengua, religión, opinión política o filosófica, pertenencia a una organización sindical, social o de derechos humanos, o para promover los intereses de cualquier partido o movimiento político o afectar los derechos y garantías de los partidos políticos de oposición.

Aunque algunos aspectos sean opinables - la finalidad de proteger los intereses económicos parece muy amplia, pudiéndose haber aclarado que tal protección tendría lugar ante amenazas externas al país- parece interesante la disposición transcripta, en el contexto latinoamericano.

También merecen cita los principios de las actividades de inteligencia y contrainteligencia establecidos en el artículo 5:

Principio de necesidad: La actividad de inteligencia y contrainteligencia debe ser necesaria para alcanzar los fines constitucionales deseados; es decir que podrá recurrirse a ésta siempre que no existan otras actividades menos lesivas que permitan alcanzar tales fines.

Principio de idoneidad: La actividad de inteligencia y contrainteligencia debe hacer uso de medios que se adecuen al logro de los fines definidos en el artículo 4 de esta Ley; es decir que se deben usar los medios aptos para el cumplimiento de tales fines y no otros.

Principio de proporcionalidad: La actividad de inteligencia y contrainteligencia deberá ser proporcional a los fines buscados y sus beneficios deben exceder las restricciones impuestas sobre otros principios y valores constitucionales. En 
particular, los medios y métodos empleados no deben ser desproporcionados frente a los fines que se busca lograr.

Estos principios, que procuran asegurar que la actividad de inteligencia sólo sea empleada ante situaciones que la hagan necesaria - evitándose su empleo como una actividad ordinaria del Estado, desentendiéndose de sus consecuencias institucionales - constituyen un aspecto auspicioso del proyecto de ley en comentario, al que debe agregarse la auspiciosa circunstancia de haberse concluido con la actividad del Departamento Administrativo de Seguridad (DAS) organismo de inteligencia civil colombiano que experimentara serios y fundados cuestionamientos.

Si bien otros aspectos del proyecto, como la debilidad de los controles externos establecidos, resulten objetables, desde el punto de vista de nuestro análisis, las indicadas tentativas de legislar la actividad de inteligencia en Colombia y establecerle límites y controles resultan de verdadero interés.

\section{Conclusiones}

Entre las características más salientes de la actividad de inteligencia en Latinoamérica, se encuentran la amplitud y frecuente indeterminación de las competencias de los organismos de inteligencia, características que incluyen a los organismos cuya actuación tiene lugar en el propio país y respecto de los propios habitantes, dado que - a diferencia de lo que sucede en la mayoría de los países desarrollados institucionalmente - ninguna diferencia se efectúa en las normas que reglan tal actividad entre los ámbitos interno y externo del país, y entre los extranjeros y los propios ciudadanos y residentes legales, hallándose habitualmente asignadas las competencias en ambos aspectos a un organismo de inteligencia único. ${ }^{25}$

Cabe destacar además que en la práctica, y pese a que habitualmente las normas relativas a la cuestión asignan a los organismos de inteligencia latinoamericanos competencias en los ámbitos externo e interno, su actividad se ejerce en la práctica casi exclusivamente en y referida al ámbito interno, incursionando frecuentemente en la política partidaria del país de que se trate.

La comprensión adecuada de la naturaleza excepcional de un instrumento como la actividad de inteligencia, dadas sus características, y de la consiguiente necesidad de reservar su empleo en el ámbito interno a las amenazas contra la seguridad del Estado y contra el sistema democrático - sin perjuicio de utilizarla con mayor amplitud en el exterior del país para escudriñar el ámbito internacional en busca, no sólo de amenazas, sino de oportunidades para la acción política y económica del país - debiera llevar

25 Remitimos en este aspecto a la visión comparativa efectuada en, UGARTE, José Manuel. El control público de la actividad de inteligencia en América Latina. 
a una definición más precisa de tales competencias en el sentido indicado, así como a asegurar su vigencia a través del control.

Cierto es, no obstante, que una diferenciación de competencias entre los ámbitos de competencia externo e interno sería de problemática realización en Latinoamérica, dado que existiendo un organismo de inteligencia civil único con competencias en ambos ámbitos, en la práctica es la situación política interna quien absorbe todos los recursos y esfuerzos. Para los países latinoamericanos y especialmente para los más importantes sería de suma utilidad contar con un organismo de inteligencia exterior - para una potencia emergente como Brasil, una necesidad, para México y para Argentina, una conveniencia fuerte - pero el peso de las estructuras y tradiciones en la materia heredadas del conflicto Este-Oeste lo hacen en la práctica difícil.

En suma, es preciso limitar la actividad de inteligencia a aquellos aspectos para los que es realmente necesaria, tanto en beneficio de la eficiencia y eficacia de esta importante actividad del Estado, como de la protección de los derechos individuales de los habitantes. 